\title{
Robust PollnSAR optimal interferogram estimation method based on generalized scattering vector
}

\author{
Zhiyong Suo ${ }^{1, *}$ and Ming $\mathrm{Li}^{2,3}$ \\ 1. National Lab of Radar Singal Processing, Xidian University, Xi’an 710071, China; \\ 2. Beijing Aerospace Automatic Control Institute, Beijing 100854, China; \\ 3. National Key Laboratory of Science and Technology on Aerospace Intelligent Control, Beijing 100854, China
}

\begin{abstract}
For the polarimetric synthetic aperture radar interferometry (PollnSAR) processing, it is necessary to coregister all the images, including the coregistration of polarimetric SAR images and the coregistration of interferometric SAR images. Otherwise, the performance of the estimated optimal interferograms will be deteriorated. A generalized scattering vector (GSV) model is proposed to execute the PollnSAR optimal interferograms estimation. The generalized scattering vector is constructed by the Pauli scattering vectors of the processing pixel and the surrounding pixels. Even though there are coregistration errors, all the polarimetric information of the current processing pixel is entirely included in the generalized scattering vector. Therefore, the GSV-based method can automatically recover the optimal scattering mechanisms of the processing pixel with coregistration errors either in interferoemetric channels or polarimetric channels. Theoretical analysis and processing results of simulated PoISARPro data and real PALSAR data validate the effectiveness and correctness of the proposed method.
\end{abstract}

Keywords: polarimetric synthetic aperture radar interferometry (PollnSAR), generalized scattering vector (GSV), optimal interferogram, coregistration error, Pauli basis.

DOI: $10.21629 /$ JSEE.2017.03.06

\section{Introduction}

Polarimetric synthetic aperture radar interferometry (PolInSAR) is widely used in different fields such as the vegetable parameters inversion $[1-5]$ and the ground target recognition and classification [6-8]. The polarimetric coherence optimization is usually utilized to obtain the optimal interferograms of different scattering mechanisms. Actually, the principle of the polarimetric coherence optimization is to find the different polarimetric state

Manuscript received July 22, 2016

*Corresponding author.

This work was supported by the National Natural Science Foundation of China (61471276; 61671355) and the Areospace T.T.\&.C. Innovation Program. combinations which correspond to the highest possible interferometric coherence. However, it is assumed that the SAR images, including the polarimetric SAR images and the interferometric SAR images, are all accurately coregistered during the PolInSAR processing. As is known that the accurate coregistration of SAR images is difficult for InSAR processing especially for high resolution SAR images, so does the PolInSAR processing. The coregistration errors will affect the optimal interferograms estimation (i.e., the optimal scattering mechanisms), mainly the noise level of the interferograms. Therefore, it can be predicted that the coregistration errors must be small enough to obtain a better performance on the optimal scattering mechanisms estimation.

The coregistration approaches used in PolInSAR processing are similar to the conventional InSAR processing. The traditional coregistration methods only use the SAR images of the same polarimetric channel (for example, the HH channel or the VV channel) to obtain the coregistration parameters and the interpolation coefficients, including the cross correlation method [9], the average fluctuation function method [10] and the maximum spectrum method $[11,12]$. The coregistration parameters and the interpolation coefficients obtained by the single channel will be used to coregister other polarimetric channels in PolInSAR processing. The polarimetric characteristics are not used in the traditional methods [9-12], so the coregistration accuracy of other polarimetric channels cannot be guaranteed except the reference channel. In order to improve the coregistration accuracy in PolInSAR, the total power image pairs of all polarimetric channels are utilized to obtain the coregistration parameters. However, in the power images the phases are lost and only the total power is left, which limits the improvement of the coregistration accuracy. The similarity parameter, representing the similar scattering mechanism, was introduced 
in [13] to improve the coregistration accuracy between the polarimetric images. In [14], the Cameron decomposition $[15,16]$ was used to obtain the same scattering mechanism, and then the conventional InSAR coregistration methods are employed to obtain the coregistration parameters. The scale invariant feature transform (SIFT) algorithm was used to select ground control points in the power image to improve the coregistration performance for the polarimetric SAR/InSAR processing in [17]. From the PolSAR/PolInSAR processing results given in [13-17], it is concluded that the higher coregistration accuracy will be obtained by using the same scattering mechanisms compared to the conventional processing.

When the interferometric SAR images are coregistered, it is still indicated that all the polarimetric SAR images are accurately coregistered. Generally, the true phase center of an antenna cannot be obtained inerrably and the polarimetric channels are not completely orthogonal to each other. Even though the polarimetric calibration is executed, there still remain residue image shift errors in different polarimetric channels. Furthermore, when the horizontal channel and the vertical channel are placed separately in the PolSAR/PolInSAR systems, the images of different polarimetric channels have to be coregistered inevitably. Thus the coregistration errors are unavoidable in different polarimetric channels. For PolInSAR processing, coregistration errors exist not only in the polarimetric SAR images, but also in the interferometric SAR images. Both coregistration errors will affect the optimal interferograms estimation. Up to now, the effect of coregistration errors of polarimetric channels on optimal interferogram estimation has not been discussed in references. In order to alleviate the coregistration errors effect, the joint pixel processing method is used to estimate InSAR phase [18,19] or suppress the clutter in SAR-GMTI [20]. The joint pixel processing method relaxes the limitation of coregistration accuracy, and has robust performance of InSAR phase estimation and clutter suppression. Based on the ideal used in InSAR or SAR-GMTI, we use the generalized scattering vector (GSV), which is constructed by the scattering vectors of the processing pixel and its neighboring pixels, to overcome the effect of the coregistration errors on robust optimal interferogram estimation.

This paper is arranged as follows. By reviewing the conventional Pauli-based method of optimal interferogram estimation, the GSV model is proposed in Section 2. In Section 3, we analyze the performance of the optimal interferogram estimation of the GSV method when there are coregistration errors either in the polarimetric channels or in the interferometric channels. The detailed steps to estimate the optimal interferograms using the GSV-based method are listed in Section 4. The performance of the pro- posed method is investigated by the PolSARPro simulated data and the real PALSAR data in Section 5. We summarize the whole paper in Section 6.

\section{Signal model of GSV}

\subsection{Pauli scattering vector and optimal interferogram estimation}

Assuming reciprocal scattering, we get the Pauli scattering vector of PolSAR [1] as

$$
\begin{gathered}
\boldsymbol{k}_{m}=\frac{1}{\sqrt{2}}\left[S_{m, h h}+S_{m, v v}, S_{m, h h}-S_{m, v v}, 2 S_{m, h v}\right]^{\mathrm{T}}, \\
m=1,2
\end{gathered}
$$

where $m=1$ represents the master interferometric channel, $m=2$ represents the slave interferometric channel, and the superscript " $T$ " represents transpose. The polarimetric coherence optimization is used to maximize the following equation:

$$
\max _{\boldsymbol{\omega}_{1}, \boldsymbol{\omega}_{2}} \xi=\frac{\boldsymbol{\omega}_{1}^{\mathrm{H}} \boldsymbol{\Omega}_{12} \boldsymbol{\omega}_{2}}{\sqrt{\boldsymbol{\omega}_{1}^{\mathrm{H}} \boldsymbol{T}_{11} \boldsymbol{\omega}_{1}} \sqrt{\boldsymbol{\omega}_{2}^{\mathrm{H}} \boldsymbol{T}_{22} \boldsymbol{\omega}_{2}}}
$$

where $\boldsymbol{\omega}_{1}$ and $\boldsymbol{\omega}_{2}$ represent different scattering mechanisms. $\boldsymbol{T}_{11}=\left\langle\boldsymbol{k}_{1} \boldsymbol{k}_{1}^{\mathrm{H}}\right\rangle$ and $\boldsymbol{T}_{22}=\left\langle\boldsymbol{k}_{2} \boldsymbol{k}_{2}^{\mathrm{H}}\right\rangle$ are the hermitian coherency matrices corresponding to master channel and slave channel, respectively. $\boldsymbol{\Omega}_{12}=\left\langle\boldsymbol{k}_{1} \boldsymbol{k}_{2}^{\mathrm{H}}\right\rangle$ is the covariance matrix containing all the polarimetric and interferometric information. From (2), we can see that the interferometric coherence is strongly dependent on the polarization states (i.e., different $\boldsymbol{\omega}_{1}$ and $\boldsymbol{\omega}_{2}$ ). To maximize (2), the complex Lagrange function [21] is used. The optimal scattering mechanism estimation can be obtained [1] by eigen-decompositing the equations:

$$
\begin{aligned}
& T_{11}^{-1} \boldsymbol{\Omega}_{12} T_{22}^{-1} \boldsymbol{\Omega}_{12}^{\mathrm{H}} \boldsymbol{\omega}_{1 j}=v_{j} \boldsymbol{\omega}_{1 j} \\
& T_{22}^{-1} \boldsymbol{\Omega}_{12}^{\mathrm{H}} T_{11}^{-1} \boldsymbol{\Omega}_{12} \boldsymbol{\omega}_{2 j}=v_{j} \boldsymbol{\omega}_{2 j}
\end{aligned}
$$

where the superscript " $H$ " represents the conjugate transpose.

The optimal coherence corresponds to the square roots of the eigenvalues $v_{j}(j=1,2,3)$, and the optimal scattering mechanisms (i.e., the optimal interferegrams) are obtained by

$$
\varphi_{i}=\arg \left(\boldsymbol{\omega}_{1 j}^{\mathrm{H}} \boldsymbol{\Omega}_{12} \boldsymbol{\omega}_{2 j}\right), \quad j=1,2,3
$$

where $\boldsymbol{\omega}_{1 j}$ and $\boldsymbol{\omega}_{2 j}$ are constrained by the following equation:

$$
\arg \left(\boldsymbol{\omega}_{1 j}^{\mathrm{H}} \boldsymbol{\omega}_{2 j}\right)=0, \quad j=1,2,3 .
$$

\subsection{GSV model}

For the conventional Pauli-based method, all the SAR images are assumed to be accurately coregistered for the optimal interferogram estimation. In fact, it is impossible 
for us to simultaneously coregister all the SAR images of the polarimetric channels and/or the interferometric channels accurately. Therefore, the GSV-based method is used to alleviate the rigorous limitation of the coregistration errors for PolInSAR processing. For the sake of simplicity, we first assume that the GSV model is constructed by two pixels of the Pauli scattering vectors, as is shown in Fig. 1.

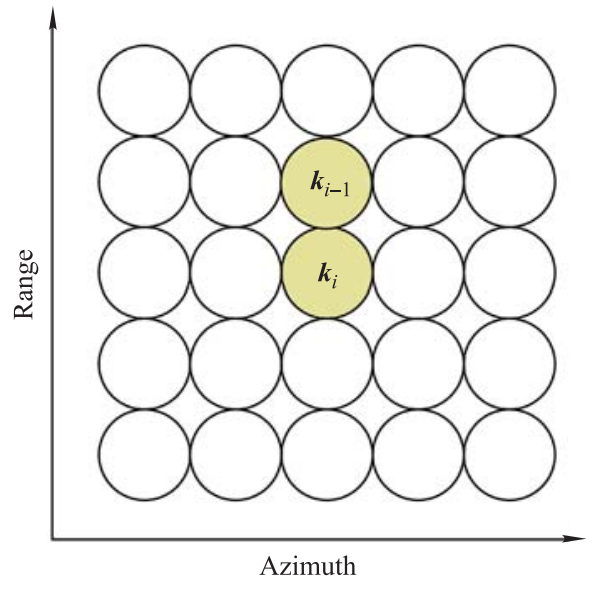

Fig. 1 Sketch of GSV construction

From Fig. 1, we can see that the scattering vectors of the processing pixel (marked as $\boldsymbol{k}_{i}$ ) and its neighboring pixel are simultaneously utilized to construct the GSV. Therefore, the GSV can be expressed as

$$
\begin{gathered}
\boldsymbol{k}_{m}^{(G)}=\left[\boldsymbol{k}_{m,(i-1)}^{\mathrm{T}} \boldsymbol{k}_{m,(i)}^{\mathrm{T}}\right]^{\mathrm{T}}= \\
\frac{1}{\sqrt{2}}\left[S_{m h h, i-1}+S_{m v v, i-1}, S_{m h h, i-1}-S_{m v v, i-1},\right. \\
\left.2 S_{m h v, i-1}, S_{m h h, i}+S_{m v v, i}, S_{m h h, i}-S_{m v v, i}, 2 S_{m h v, i}\right]^{\mathrm{T}}
\end{gathered}
$$

where $m=1,2$ have the same meaning as (1).

If the pixels of polarimetric SAR images are independent, the interferometric coherence exists only in corresponding pixel pairs, i.e.,

$$
\begin{gathered}
\boldsymbol{k}_{1}(i) \boldsymbol{k}_{2}^{\mathrm{H}}(j)= \begin{cases}\boldsymbol{\Omega}_{12}, & i=j \\
\mathbf{0}_{3 \times 3}, & i \neq j\end{cases} \\
\boldsymbol{k}_{m}(i) \boldsymbol{k}_{m}^{\mathrm{H}}(j)= \begin{cases}\boldsymbol{T}_{m m}, & i=j \\
\mathbf{0}_{3 \times 3}, & i \neq j\end{cases}
\end{gathered}
$$

where $m=1,2$.

Similar to the conventional Pauli-based method processing, the GSV-based optimal scattering mechanisms can be obtained as

$$
\begin{aligned}
& \boldsymbol{T}_{11}^{(G)-1} \boldsymbol{\Omega}_{12}^{(G)} \boldsymbol{T}_{22}^{(G)-1} \boldsymbol{\Omega}_{12}^{(G) \mathrm{H}} \boldsymbol{\omega}_{1}^{(G)}=\boldsymbol{v}_{1}^{(G)} \boldsymbol{\omega}_{1}^{(G)} \\
& \boldsymbol{T}_{22}^{(G)-1} \boldsymbol{\Omega}_{12}^{(G) \mathrm{H}} \boldsymbol{T}_{11}^{(G)-1} \boldsymbol{\Omega}_{12}^{(G)} \boldsymbol{\omega}_{2}^{(G)}=\boldsymbol{v}_{2}^{(G)} \boldsymbol{\omega}_{2}^{(G)}
\end{aligned}
$$

where the superscript " $(G)$ " represents the GSV-based processing. The two matrices $\boldsymbol{T}_{11}^{(G)-1} \boldsymbol{\Omega}_{12}^{(G)} \boldsymbol{T}_{22}^{(G)-1} \boldsymbol{\Omega}_{12}^{(G) \mathrm{H}}$ and $\boldsymbol{T}_{22}^{(G)-1} \boldsymbol{\Omega}_{12}^{(G) \mathrm{H}} \boldsymbol{T}_{11}^{(G)-1} \boldsymbol{\Omega}_{12}^{(G)}$ are named as "coefficient matrices".

$$
\begin{aligned}
& \boldsymbol{\Omega}_{12}^{(G)}=\boldsymbol{k}_{1}^{(G)} \boldsymbol{k}_{2}^{(G) \mathrm{H}}=\left[\begin{array}{cc}
\boldsymbol{\Omega}_{12,(i-1, i-1)} & \mathbf{0} \\
\mathbf{0} & \boldsymbol{\Omega}_{12,(i, i)}
\end{array}\right] \\
& \boldsymbol{T}_{m m}^{(G)}=\boldsymbol{k}_{m}^{(G)} \boldsymbol{k}_{m}^{(G) \mathrm{H}}=\left[\begin{array}{cc}
\boldsymbol{T}_{m m,(i-1, i-1)} & \mathbf{0} \\
\mathbf{0} & \boldsymbol{T}_{m m,(i, i)}
\end{array}\right]
\end{aligned}
$$

In the following, we analyze the relationship of the eigenvalues and the eigenvectors between (3) and (10).

The coefficient matrix of (10) can be rewritten as follows:

$$
\begin{gathered}
\boldsymbol{T}_{11}^{(G)-1} \boldsymbol{\Omega}_{12}^{(G)} \boldsymbol{T}_{22}^{(G)-1} \boldsymbol{\Omega}_{12}^{(G) \mathrm{H}}= \\
{\left[\begin{array}{cc}
\boldsymbol{T}_{11,(i-1, i-1)}^{-1} & \mathbf{0} \\
\mathbf{0} & \boldsymbol{T}_{11,(i, i)}^{-1}
\end{array}\right]\left[\begin{array}{cc}
\boldsymbol{\Omega}_{12,(i-1, i-1)} & \mathbf{0} \\
\mathbf{0} & \boldsymbol{\Omega}_{12,(i, i)}
\end{array}\right]} \\
{\left[\begin{array}{cc}
\boldsymbol{T}_{22,(i-1, i-1)}^{-1} & \mathbf{0} \\
\mathbf{0} & \boldsymbol{T}_{22,(i, i)}^{-1}
\end{array}\right]\left[\begin{array}{cc}
\boldsymbol{\Omega}_{12,(i-1, i-1)}^{\mathrm{H}} & \mathbf{0} \\
\mathbf{0} & \boldsymbol{\Omega}_{12,(i, i)}^{\mathrm{H}}
\end{array}\right]=} \\
{\left[\begin{array}{c}
\boldsymbol{T}_{11,(i-1, i-1)}^{-1} \boldsymbol{\Omega}_{12,(i-1, i-1)} \boldsymbol{T}_{22,(i-1, i-1)}^{-1} \boldsymbol{\Omega}_{12,(i-1, i-1)}^{\mathrm{H}} \\
\mathbf{0} \\
\mathbf{0} \\
\boldsymbol{T}_{11,(i, i)}^{-1} \boldsymbol{\Omega}_{12,(i, i)} \boldsymbol{T}_{22,(i, i)}^{-1} \boldsymbol{\Omega}_{12,(i, i)}^{\mathrm{H}}
\end{array}\right] .}
\end{gathered}
$$

If the scattering vectors of the neighboring pixels are identical independent distribution (i.i.d) samples, we have

$$
\begin{aligned}
\left\langle\boldsymbol{T}_{11,(i-1, i-1)}\right\rangle & =\left\langle\boldsymbol{T}_{11,(i, i)}\right\rangle \\
\left\langle\boldsymbol{T}_{22,(i-1, i-1)}\right\rangle & =\left\langle\boldsymbol{T}_{22,(i, i)}\right\rangle \\
\left\langle\boldsymbol{\Omega}_{12,(i-1, i-1)}\right\rangle & =\left\langle\boldsymbol{\Omega}_{12,(i, i)}\right\rangle
\end{aligned}
$$

where $\langle\cdot\rangle$ means the expectation. Therefore, the expectations of the diagonal block matrices in (14) are equal, i.e.,

$$
\begin{gathered}
\left\langle\boldsymbol{T}_{11,(i-1, i-1)}^{-1} \boldsymbol{\Omega}_{12,(i-1, i-1)} \boldsymbol{T}_{22,(i-1, i-1)}^{-1} \boldsymbol{\Omega}_{12,(i-1, i-1)}^{\mathrm{H}}\right\rangle= \\
\left\langle\boldsymbol{T}_{11,(i, i)}^{-1} \boldsymbol{\Omega}_{12,(i, i)} \boldsymbol{T}_{22,(i, i)}^{-1} \boldsymbol{\Omega}_{12,(i, i)}^{\mathrm{H}}\right\rangle=\boldsymbol{A} .
\end{gathered}
$$

Using (18), we can rewrite (14) as

$$
\boldsymbol{T}_{11}^{(G)-1} \boldsymbol{\Omega}_{12}^{(G)} \boldsymbol{T}_{22}^{(G)-1} \boldsymbol{\Omega}_{12}^{(G) \mathrm{H}}=\boldsymbol{I}_{2 \times 2} \otimes \boldsymbol{A}
$$

where " $\otimes$ " means the Kronecker product.

Similar to the analysis of (10), (11) can be rewritten as

$$
\begin{gathered}
\boldsymbol{T}_{22}^{(G)-1} \boldsymbol{\Omega}_{12}^{(G) \mathrm{H}} \boldsymbol{T}_{11}^{(G)-1} \boldsymbol{\Omega}_{12}^{(G)}= \\
{\left[\begin{array}{c}
\boldsymbol{T}_{22,(i-1, i-1)}^{-1} \boldsymbol{\Omega}_{12,(i-1, i-1)}^{\mathrm{H}} \boldsymbol{T}_{11,(i-1, i-1)}^{-1} \boldsymbol{\Omega}_{12,(i-1, i-1)} \\
\mathbf{0} \\
\boldsymbol{T}_{22,(i, i)}^{-1} \boldsymbol{\Omega}_{12,(i, i)}^{\mathrm{H}} \boldsymbol{T}_{11,(i, i)}^{-1} \boldsymbol{\Omega}_{12,(i, i)}
\end{array}\right]=\boldsymbol{I}_{2 \times 2} \otimes \boldsymbol{B}}
\end{gathered}
$$


where $\boldsymbol{B}=\boldsymbol{T}_{22,(i, i)}^{-1} \boldsymbol{\Omega}_{12,(i, i)}^{\mathrm{H}} \boldsymbol{T}_{11,(i, i)}^{-1} \boldsymbol{\Omega}_{12,(i, i)}$.

Theorem 1 [18] If $\lambda(\boldsymbol{P})$ and $\lambda(\boldsymbol{Q})$ are the eigenvalues of the matrices $\boldsymbol{P}$ and $\boldsymbol{Q}$ respectively, and $\boldsymbol{u}(\boldsymbol{P})$ and $\boldsymbol{u}(\boldsymbol{Q})$ are the corresponding eigenvectors respectively, then $\lambda(\boldsymbol{P}) \lambda(\boldsymbol{Q})$ are the eigenvalues of the matrix $\boldsymbol{P} \otimes$ $\boldsymbol{Q}$, and $\boldsymbol{u}(\boldsymbol{P}) \otimes \boldsymbol{u}(\boldsymbol{Q})$ are the corresponding vectors of $\lambda(\boldsymbol{P}) \lambda(\boldsymbol{Q})$.

According to Theorem 1, (19) has three different eigenvalues, and each eigenvalue is a two-fold eigenvalue.

The optimal scattering mechanisms (i.e., the optimal interferograms) are obtained by

$$
\varphi_{i}=\arg \left(\sum_{k=1}^{2} \lambda_{i, k} \boldsymbol{\omega}_{i, k}^{(G) \mathrm{H}} \boldsymbol{\Omega}_{12}^{(G)} \boldsymbol{\omega}_{i, k}^{(G)}\right), \quad i=1,2,3 .
$$

\section{Robustness of GSV to coregistration errors}

Without loss of generality and for simplicity, we first assume that coregistration errors exist either in the slave polarimetric channels or in the slave interferometric channels. Furthermore, we assume that the coregistration errors are only in one direction and the SAR images of master antenna are accurately coregistered.

Theorem 2 [22] The eigenspectrum (i.e., the distribution of eigenvalues) of a covariance matrix is invariant no matter how the elements of the corresponding data vector are permuted.

(i) Accurate coregistration

For accurate coregistration, the optimal scattering mechanisms can be obtained by (21).

(ii) Coregistration errors of interferometric images

When coregistration errors are in the slave interferometric PolSAR images, the Pauli scattering vector of the slave polarimetric SAR images corresponding to the master polarimetric SAR images can be expressed as

$$
\boldsymbol{k}_{2,(i)}=\boldsymbol{k}_{2,(i)}^{(a)}+\boldsymbol{k}_{2,(i+1)}^{(b)}
$$

where $\boldsymbol{k}_{2,(i+1)}^{(b)}$ is the signal mixed into the processing pixel in the slave channel. When there are coregistration errors, the GSV can be constructed as Fig. 2.

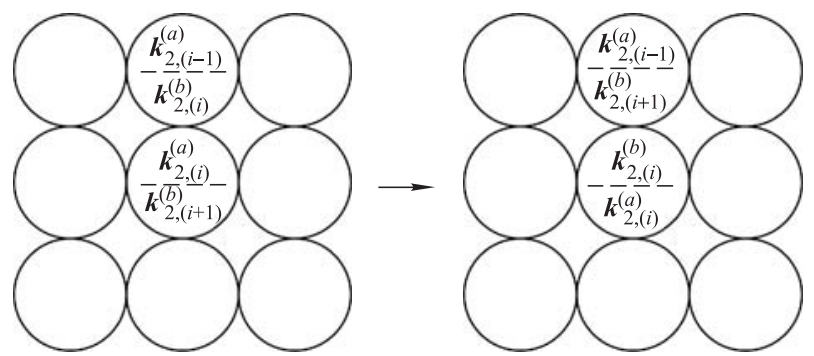

Fig. 2 Construction of GSV of slave PolSAR images with coregistration errors of interferometric images
As is shown in Fig. 2, when there are only coregistration errors between interferometric channels, the GSV can be expressed as

$$
\boldsymbol{k}_{2}^{(G)}=\left[\boldsymbol{k}_{2,(i-1)}^{(a) \mathrm{T}}+\boldsymbol{k}_{2,(i)}^{(b) \mathrm{T}}, \boldsymbol{k}_{2,(i)}^{(a) \mathrm{T}}+\boldsymbol{k}_{2,(i+1)}^{(b) \mathrm{T}}\right]^{\mathrm{T}} .
$$

Using Theorem 2 and rearranging the Pauli scattering vectors as Fig. 3, $\boldsymbol{k}_{2}^{(G)}$ in (23) can be rewritten as

$$
\boldsymbol{k}_{2}^{(G)}=\left[\boldsymbol{k}_{2,(i-1)}^{(a) \mathrm{T}}+\boldsymbol{k}_{2,(i+1)}^{(b) \mathrm{T}}, \boldsymbol{k}_{2,(i)}^{\mathrm{T}}\right]^{\mathrm{T}}
$$

where $\boldsymbol{k}_{2,(i-1)}^{(a)}, \boldsymbol{k}_{2,(i+1)}^{(b)}, \boldsymbol{k}_{2,(i)}$ are independent to each other, so we have

$$
\begin{gathered}
\boldsymbol{k}_{2,(i-1)}^{(a)} \boldsymbol{k}_{2,(i)}^{\mathrm{H}}=\boldsymbol{T}_{22,(i-1, i)}=\mathbf{0}_{3 \times 3} \\
\boldsymbol{k}_{2,(i+1)}^{(b)} \boldsymbol{k}_{2,(i)}^{\mathrm{H}}=\boldsymbol{T}_{22,(i+1, i)}=\mathbf{0}_{3 \times 3} \\
\boldsymbol{k}_{2,(i-1)}^{(a)} \boldsymbol{k}_{2,(i+1)}^{(b) \mathrm{H}}=\boldsymbol{T}_{22,(i-1, i+1)}=\mathbf{0}_{3 \times 3} .
\end{gathered}
$$

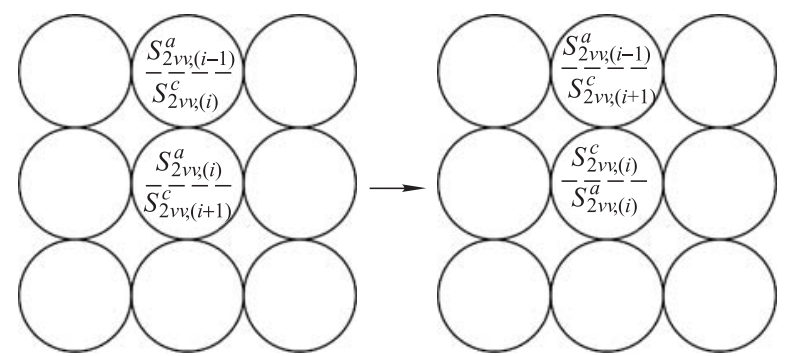

Fig. 3 Sketch of pixels rearrangement with polarimetric coregistration errors

Furthermore, the Pauli scattering vector of master channels can be expressed as $\boldsymbol{k}_{1,(i-1)}=\boldsymbol{k}_{1,(i-1)}^{(a)}+\boldsymbol{k}_{1,(i-1)}^{(b)}$, then we have

$$
\begin{gathered}
\boldsymbol{k}_{1,(i-1)}^{(a)} \boldsymbol{k}_{2,(i+1)}^{(b) \mathrm{H}}=\boldsymbol{\Omega}_{12,(i-1, i+1)}^{(a)(b)}=\mathbf{0}_{3 \times 3} \\
\boldsymbol{k}_{1,(i-1)}^{(b)} \boldsymbol{k}_{2,(i+1)}^{(b) \mathrm{H}}=\boldsymbol{\Omega}_{12,(i-1, i+1)}^{(b)}=\mathbf{0}_{3 \times 3} .
\end{gathered}
$$

Using relations of (25) - (29) and the similar deduction of (10), the coefficient matrix with coregistration errors of interferometric channels can be expressed as

$$
\begin{gathered}
\boldsymbol{T}_{11}^{(G)-1} \boldsymbol{\Omega}_{12}^{(G)} \boldsymbol{T}_{22}^{(G)-1} \boldsymbol{\Omega}_{12}^{(G) \mathrm{H}}= \\
{\left[\begin{array}{cc}
\boldsymbol{T}_{11,(i-1, i-1)}^{-1} & \mathbf{0} \\
\mathbf{0} & \boldsymbol{T}_{11,(i, i)}^{-1}
\end{array}\right]\left[\begin{array}{cc}
\boldsymbol{\Omega}_{12,(i-1, i-1)}^{(a)} & \mathbf{0} \\
\mathbf{0} & \boldsymbol{\Omega}_{12,(i, i)}
\end{array}\right]} \\
{\left[\begin{array}{cc}
\boldsymbol{T}_{22,(i-1, i-1)}^{(a)}+\boldsymbol{T}_{22,(i+1, i+1)}^{(b)} & \mathbf{0} \\
\mathbf{0} & \boldsymbol{T}_{22,(i, i)}
\end{array}\right]} \\
{\left[\begin{array}{cc}
\boldsymbol{\Omega}_{12,(i-1, i-1)}^{(a) \mathrm{H}} & \mathbf{0} \\
\mathbf{0} & \boldsymbol{\Omega}_{12,(i, i)}^{\mathrm{H}}
\end{array}\right]}
\end{gathered}
$$


Theorem 3 [23] Supposing $\boldsymbol{A}$ is an $n \times n$ invertible matrix, and $\boldsymbol{x}, \boldsymbol{y}$ are both $n \times 1$ vectors, if $\boldsymbol{A}+\boldsymbol{x} \boldsymbol{y}^{\mathrm{H}}$ is invertible, then we have

$$
\left(\boldsymbol{A}+\boldsymbol{x} \boldsymbol{y}^{\mathrm{H}}\right)^{-1}=\boldsymbol{A}^{-1}-\frac{\boldsymbol{A}^{-1} \boldsymbol{x} \boldsymbol{y}^{\mathrm{H}} \boldsymbol{A}^{-1}}{1+\boldsymbol{y}^{\mathrm{H}} \boldsymbol{A}^{-1} \boldsymbol{x}} .
$$

According to Theorem 3, (30) can be deduced as

$$
\begin{gathered}
{\left[\boldsymbol{T}_{11}^{(G)}\right]^{-1}\left[\boldsymbol{\Omega}_{12}^{(G)}\right]\left[\boldsymbol{T}_{22}^{(G)}\right]^{-1}\left[\boldsymbol{\Omega}_{12}^{(G)}\right]^{\mathrm{H}}=} \\
{\left[\begin{array}{cc}
\boldsymbol{T}_{11,(i-1, i-1)}^{-1} \boldsymbol{\Omega}_{12,(i-1, i-1)}^{(a)}\left(\boldsymbol{T}_{22,(i-1, i-1)}^{(a)}+\boldsymbol{k}_{2,(i+1)}^{(b)} \boldsymbol{k}_{2,(i+1)}^{(b) \mathrm{H}}\right)^{-1} \boldsymbol{\Omega}_{12,(i-1, i-1)}^{(a) \mathrm{H}} & \boldsymbol{T}_{11,(i, i)}^{-1} \boldsymbol{\Omega}_{12,(i, i)} \boldsymbol{T}_{22,(i, i)}^{-1} \boldsymbol{\Omega}_{12,(i, i)}^{\mathrm{H}}
\end{array}\right]=} \\
\mathbf{0} \\
{\left[\begin{array}{ccc}
\boldsymbol{T}_{11,(i-1, i-1)}^{-1} \boldsymbol{\Omega}_{12,(i-1, i-1)}^{(a)} \boldsymbol{T}_{22,(i-1, i-1)}^{(a)-1} \boldsymbol{\Omega}_{12,(i-1, i-1)}^{(a) \mathrm{H}} \\
\mathbf{0} & \boldsymbol{T}_{11,(i, i)}^{-1} \boldsymbol{\Omega}_{12,(i, i)} \boldsymbol{T}_{22,(i, i)}^{-1} \boldsymbol{\Omega}_{12,(i, i)}^{\mathrm{H}}
\end{array}\right]-\left[\begin{array}{cc}
\Delta \boldsymbol{A}_{1} & \mathbf{0} \\
\mathbf{0} & \mathbf{0}
\end{array}\right]}
\end{gathered}
$$

where

$$
\begin{gathered}
\Delta A_{1}=\frac{\boldsymbol{T}_{11,(i-1, i-1)}^{-1} \Omega_{12,(i-1, i-1)}^{(a)} \boldsymbol{T}_{22,(i-1, i-1)}^{(a)-1}}{1+\boldsymbol{k}_{2,(i+1)}^{(b) \mathrm{H}} \boldsymbol{T}_{22,(i-1, i-1)}^{(a)-1} \boldsymbol{k}_{2,(i+1)}^{(b)}} . \\
\boldsymbol{T}_{22,(i+1, i+1)}^{(b)} \boldsymbol{T}_{22,(i-1, i-1)}^{(a)-1} \boldsymbol{\Omega}_{12,(i-1, i-1)}^{(a) \mathrm{H}} .
\end{gathered}
$$

If the used pixels are i.i.d samples, then

$$
\left\langle\boldsymbol{T}_{22,(i+1, i+1)}^{(b)}\right\rangle=\left\langle\boldsymbol{T}_{22,(i-1, i-1)}^{(b)}\right\rangle .
$$

The superscript "(b)" represents the signal introduced by the coregistration errors of the slave images. For the conventional InSAR processing, the coregistration accuracy is less than $1 / 10$ pixels, so the power of part " $(b)$ " is far less than the power of part " $(a)$ ", i.e.,

$$
\operatorname{tr}\left(\boldsymbol{T}_{22,(i-1, i-1)}^{(b)}\right) \ll \operatorname{tr}\left(\boldsymbol{T}_{22,(i-1, i-1)}^{(a)}\right) .
$$

$\boldsymbol{T}_{22,(i-1, i-1)}^{(a)-1}$ is a Hermitian and non-negative matrix, so the quadratic function

$$
\boldsymbol{k}_{2,(i+1)}^{(b) \mathrm{H}} \boldsymbol{T}_{22,(i-1, i-1)}^{(a)-1} \boldsymbol{k}_{2,(i+1)}^{(b)} \geqslant 0 .
$$

Therefore, we have

$$
\begin{gathered}
\operatorname{tr}\left(\Delta \boldsymbol{A}_{1}\right) \ll \operatorname{tr}\left(\frac{\boldsymbol{T}_{11,(i-1, i-1)}^{-1} \boldsymbol{\Omega}_{12,(i-1, i-1)}^{(a)} \boldsymbol{T}_{22,(i-1, i-1)}^{(a)-1}}{1+\boldsymbol{k}_{2,(i+1)}^{(b) \mathrm{H}} \boldsymbol{T}_{22,(i-1, i-1)}^{(a)-1} \boldsymbol{k}_{2,(i+1)}^{(b)}} .\right. \\
\operatorname{tr}\left(\frac{\boldsymbol{T}_{11,(i-1, i-1)}^{-1} \boldsymbol{\Omega}_{12,(i-1, i-1)}^{(a)} \boldsymbol{T}_{22,(i-1, i-1)}^{(a)-1} \boldsymbol{\Omega}_{12,(i-1, i-1)}^{(a) \mathrm{H}}}{1+\boldsymbol{k}_{2,(i+1)}^{(b) \mathrm{H}} \boldsymbol{T}_{22,(i-1, i-1)}^{(a)-1} \boldsymbol{k}_{2,(i+1)}^{(b)}}\right) \leqslant \\
\operatorname{tr}\left(\boldsymbol{T}_{11,(i-1, i-1)}^{-1} \boldsymbol{\Omega}_{12,(i-1, i-1)}^{(a)} \boldsymbol{T}_{22,(i-1, i-1)}^{(a)-1} .\right. \\
\left.\boldsymbol{\Omega}_{12,(i-1, i-1)}^{(a) \mathrm{H}}\right)<\operatorname{tr}\left(\boldsymbol{T}_{11,(i-1, i-1)}^{-1} \cdot\right. \\
\left.\boldsymbol{\Omega}_{12,(i-1, i-1)} \boldsymbol{T}_{22,(i-1, i-1)}^{-1} \boldsymbol{\Omega}_{12,(i-1, i-1)}^{\mathrm{H}}\right) .
\end{gathered}
$$

Furthermore, the following term can be written as

$$
\begin{aligned}
& \boldsymbol{T}_{11,(i-1, i-1)}^{-1} \boldsymbol{\Omega}_{12,(i-1, i-1)}^{(a)} \boldsymbol{T}_{22,(i-1, i-1)}^{(a)-1} \boldsymbol{\Omega}_{12,(i-1, i-1)}^{(a) \mathrm{H}}= \\
& \boldsymbol{T}_{11,(i-1, i-1)}^{-1} \boldsymbol{\Omega}_{12,(i-1, i-1)} \boldsymbol{T}_{22,(i-1, i-1)}^{-1} \boldsymbol{\Omega}_{12,(i-1, i-1)}^{\mathrm{H}}+
\end{aligned}
$$$$
\Delta \boldsymbol{A}_{2}
$$

where

$$
\begin{gathered}
\Delta A_{2}= \\
-\boldsymbol{T}_{11,(i-1, i-1)}^{-1} \boldsymbol{\Omega}_{12,(i-1, i-1)} \boldsymbol{T}_{22,(i-1, i-1)}^{-1} \Omega_{12,(i-1, i-1)}^{(b) \mathrm{H}}+ \\
\boldsymbol{T}_{11,(i-1, i-1)}^{-1} \boldsymbol{\Omega}_{12,(i-1, i-1)}^{(b)} \\
\frac{\boldsymbol{T}_{22,(i-1, i-1)}^{-1} \boldsymbol{T}_{22,(i-1, i-1)}^{(b)} \boldsymbol{T}_{22,(i-1, i-1)}^{-1} \boldsymbol{\Omega}_{12,(i-1, i-1)}^{(a) \mathrm{H}}-}{1+\boldsymbol{k}_{2,(i-1)}^{(b) \mathrm{H}} \boldsymbol{T}_{22,(i-1, i-1)}^{-1} \boldsymbol{k}_{2,(i-1)}^{(b)}} \\
\boldsymbol{T}_{11,(i-1, i-1)}^{-1} \boldsymbol{\Omega}_{12,(i-1, i-1)}^{(b)} \boldsymbol{T}_{22,(i-1, i-1)}^{-1} \boldsymbol{\Omega}_{12,(i-1, i-1)}^{(a) \mathrm{H}}- \\
\boldsymbol{T}_{11,(i-1, i-1)}^{-1} \boldsymbol{\Omega}_{12,(i-1, i-1)} \cdot \\
\frac{\boldsymbol{T}_{22,(i-1, i-1)}^{-1} \boldsymbol{T}_{22,(i-1, i-1)}^{(b)} \boldsymbol{T}_{22,(i-1, i-1)}^{-1}}{1+\boldsymbol{k}_{2,(i-1)}^{(b) \mathrm{H}} \boldsymbol{T}_{22,(i-1, i-1)}^{-1} \boldsymbol{k}_{2,(i-1)}^{(b)}} \boldsymbol{\Omega}_{12,(i-1, i-1)}^{(a) \mathrm{H}} .
\end{gathered}
$$

Using the Theorem 3 and the deduction of (34)-(37), we have

$$
\begin{gathered}
\operatorname{tr}\left(\Delta \boldsymbol{A}_{2}\right) \ll \operatorname{tr}\left(\boldsymbol{T}_{11,(i-1, i-1)}^{-1} \boldsymbol{\Omega}_{12,(i-1, i-1)} .\right. \\
\left.\boldsymbol{T}_{22,(i-1, i-1)}^{-1} \boldsymbol{\Omega}_{12,(i-1, i-1)}^{\mathrm{H}}\right) .
\end{gathered}
$$

Then (32) can be rewritten as

$$
\begin{gathered}
\boldsymbol{T}_{11}^{(G)-1} \boldsymbol{\Omega}_{12}^{(G)} \boldsymbol{T}_{22}^{(G)-1} \boldsymbol{\Omega}_{12}^{(G) \mathrm{H}}= \\
\boldsymbol{I}_{2 \times 2} \otimes \boldsymbol{A}-\left[\begin{array}{cc}
\Delta \boldsymbol{A}_{1} & \mathbf{0} \\
\mathbf{0} & \mathbf{0}
\end{array}\right]+\left[\begin{array}{cc}
\Delta \boldsymbol{A}_{2} & \mathbf{0} \\
\mathbf{0} & \mathbf{0}
\end{array}\right] .
\end{gathered}
$$

From (37) and (40), we know that the powers of the last two terms of (41) are far less than the useful signal of $\boldsymbol{I}_{2 \times 2} \otimes \boldsymbol{A}$. Therefore, the eigenvalues of $\Delta \boldsymbol{A}_{1}$ and $\Delta A_{2}$ are far less than those of $A$, and the eigenvectors 
of $\boldsymbol{I}_{2 \times 2} \otimes \boldsymbol{A}$ will be slightly influenced, which can be almost ignored, when coregistration errors are less than 1/10 pixels. Furthremore, the biases can be calibrated with auxiliary data, like the corner reflector and/or the bare soil, etc. When there are coregistration errors, the conventional Pauli-based scattering vector cannot fully recover the optimal interferograms. On the contrary, the GSV-based processing with biases calibration is robust to coregistration errors for the optimal scattering mechanisms estimation.

Similar to the analysis of (30), we have

$$
\begin{gathered}
\boldsymbol{T}_{22}^{(G)-1} \boldsymbol{\Omega}_{12}^{(G) \mathrm{H}} \boldsymbol{T}_{11}^{(G)-1} \boldsymbol{\Omega}_{12}^{(G)}= \\
\boldsymbol{I}_{2 \times 2} \otimes \boldsymbol{B}-\left[\begin{array}{cc}
\Delta \boldsymbol{B}_{1} & \mathbf{0} \\
\mathbf{0} & \mathbf{0}
\end{array}\right]+\left[\begin{array}{cc}
\Delta \boldsymbol{B}_{2} & \mathbf{0} \\
\mathbf{0} & \mathbf{0}
\end{array}\right]
\end{gathered}
$$

where

$$
\begin{gathered}
\Delta \boldsymbol{B}_{1}=\frac{T_{22,(i-1, i-1)}^{(a)-1} T_{22,(i+1, i+1)}^{(b)} T_{22,(i-1, i-1)}^{(a)-1}}{1+\boldsymbol{k}_{2,(i+1)}^{(b) \mathrm{H}} \boldsymbol{T}_{22,(i-1, i-1)}^{(a)-1} \boldsymbol{k}_{2,(i+1)}^{(b)}} . \\
\boldsymbol{\Omega}_{12,(i-1, i-1)}^{(a)} T_{11,(i-1, i-1)}^{-1} \boldsymbol{\Omega}_{12,(i-1, i-1)}^{(a) \mathrm{H}} \\
\Delta \boldsymbol{B}_{2}= \\
-\boldsymbol{T}_{22,(i-1, i-1)}^{-1} \boldsymbol{\Omega}_{12,(i-1, i-1)} \boldsymbol{T}_{11,(i-1, i-1)}^{-1} \boldsymbol{\Omega}_{12,(i-1, i-1)}^{(b) \mathrm{H}}+ \\
\frac{\boldsymbol{T}_{22,(i-1, i-1)}^{-1} \boldsymbol{T}_{22,(i-1, i-1)}^{(b)} \boldsymbol{T}_{22,(i-1, i-1)}^{-1} \boldsymbol{\Omega}_{12,(i-1, i-1)}^{(b)}}{1+\boldsymbol{k}_{2,(i-1)}^{(b) \mathrm{H}} \boldsymbol{T}_{22,(i-1, i-1)}^{-1} \boldsymbol{k}_{2,(i-1)}^{(b)}} \\
\boldsymbol{T}_{11,(i-1, i-1)}^{-1} \boldsymbol{\Omega}_{12,(i-1, i-1)}^{(a) \mathrm{H}}- \\
\boldsymbol{T}_{22,(i-1, i-1)}^{(b)} \boldsymbol{T}_{12,(i-1, i-1)}^{-1} \boldsymbol{T}_{11,(i-1, i-1)}^{-1} \boldsymbol{\Omega}_{12,(i-1, i-1)}^{(a) \mathrm{H}}- \\
\frac{\boldsymbol{T}_{22,(i-1, i-1)}^{-1} \boldsymbol{T}_{22,(i-1, i-1)}^{(b)} \boldsymbol{T}_{22,(i-1, i-1)}^{-1} \boldsymbol{\Omega}_{12,(i-1, i-1)}}{1+\boldsymbol{k}_{2,(i-1)}^{(b) \mathrm{H}} \boldsymbol{T}_{22,(i-1, i-1)}^{-1} \boldsymbol{k}_{2,(i-1)}^{(b)}} \\
\boldsymbol{T}_{11,(i-1, i-1)}^{-1} \boldsymbol{\Omega}_{12,(i-1, i-1)}^{(a) \mathrm{H}} \cdot
\end{gathered}
$$

(iii) Coregistration errors of polarimetric images

Taking the $\mathrm{HH}$ polarization as a reference, the offsets of VV image and $\mathrm{HV}$ image relative to $\mathrm{HH}$ are $c_{1}$ and $c_{2}$, respectively. Therefore, the scattering vector of pixel $i$ with polarimetric channel coregistration errors can be expressed as

$$
\begin{gathered}
\boldsymbol{k}_{2,(i)}=\boldsymbol{k}_{2,(i)}^{(a)}+\boldsymbol{k}_{2,(i+1)}^{(c)}= \\
\frac{1}{\sqrt{2}}\left[S_{2 h h,(i)}+S_{2 v v,(i)}^{\left(a_{1}\right)}, S_{2 h h,(i)}-S_{2 v v,(i)}^{\left(a_{1}\right)}, 2 S_{2 h v,(i)}^{\left(a_{2}\right)}\right]^{\mathrm{T}}+ \\
\frac{1}{\sqrt{2}}\left[S_{2 v v,(i+1)}^{\left(c_{1}\right)},-S_{2 v v,(i+1)}^{\left(c_{1}\right)}, 2 S_{2 h v,(i+1)}^{\left(c_{2}\right)}\right]^{\mathrm{T}}
\end{gathered}
$$

where $\boldsymbol{k}_{2,(i)}^{(a)}$ is the signal corresponding to the master image, $\boldsymbol{k}_{2,(i+1)}^{(c)}$ is the signal introduced by coregistration errors. Without loss of generality, we suppose that $c_{1}=$ $c_{2}=c$ in (45).

The generalized scattering vector can be constructed as follows:

$$
\boldsymbol{k}_{2}^{(G)}=\left[\boldsymbol{k}_{2,(i-1)}^{(a) \mathrm{T}}+\boldsymbol{k}_{2,(i)}^{(c) \mathrm{T}}, \boldsymbol{k}_{2,(i)}^{(a) \mathrm{T}}+\boldsymbol{k}_{2,(i+1)}^{(c) \mathrm{T}}\right]^{\mathrm{T}} .
$$

Using Theorem 1 and Fig. 3, the rearranged generalized scattering vector is

$$
\boldsymbol{k}_{2}^{(G)}=\left[\boldsymbol{k}_{2,(i-1)}^{(a) \mathrm{T}}+\boldsymbol{k}_{2,(i+1)}^{(c) \mathrm{T}}, \boldsymbol{k}_{2,(i)}^{\mathrm{T}}\right]^{\mathrm{T}} .
$$

Similar to the analysis in the subsection (ii), the scattering vectors $\boldsymbol{k}_{2,(i-1)}^{(a) \mathrm{T}}, \boldsymbol{k}_{2,(i+1)}^{(c) \mathrm{T}}$ and $\boldsymbol{k}_{2,(i)}^{\mathrm{T}}$ are independent to each other. We can obtain the following equations when there are polarimetric channel coregistration errors:

$$
\begin{gathered}
\boldsymbol{T}_{11}^{(G)-1} \boldsymbol{\Omega}_{12}^{(G)} \boldsymbol{T}_{22}^{(G)-1} \boldsymbol{\Omega}_{12}^{(G) \mathrm{H}}= \\
\boldsymbol{I}_{2 \times 2} \otimes \boldsymbol{A}-\left[\begin{array}{cc}
\Delta \boldsymbol{A}_{3} & \mathbf{0} \\
\mathbf{0} & \mathbf{0}
\end{array}\right]+\left[\begin{array}{cc}
\Delta \boldsymbol{A}_{4} & \mathbf{0} \\
\mathbf{0} & \mathbf{0}
\end{array}\right] \\
\boldsymbol{T}_{22}^{(G)-1} \boldsymbol{\Omega}_{12}^{(G) \mathrm{H}} \boldsymbol{T}_{11}^{(G)-1} \boldsymbol{\Omega}_{12}^{(G)}= \\
\boldsymbol{I}_{2 \times 2} \otimes \boldsymbol{B}-\left[\begin{array}{cc}
\Delta \boldsymbol{B}_{3} & \mathbf{0} \\
\mathbf{0} & \mathbf{0}
\end{array}\right]+\left[\begin{array}{cc}
\Delta \boldsymbol{B}_{4} & \mathbf{0} \\
\mathbf{0} & \mathbf{0}
\end{array}\right]
\end{gathered}
$$

where

$$
\begin{aligned}
& \Delta A_{3}=\frac{\boldsymbol{T}_{22,(i-1, i-1)}^{-1} \Omega_{12,(i-1, i-1)}^{(a)} \boldsymbol{T}_{11,(i-1, i-1)}^{(a)-1}}{1+\boldsymbol{k}_{1,(i+1)}^{(c) \mathrm{H}} \boldsymbol{T}_{11,(i-1, i-1)}^{(a)-1} \boldsymbol{k}_{1,(i+1)}^{(c)}} . \\
& \boldsymbol{T}_{11,(i+1, i+1)}^{(c)} \boldsymbol{T}_{11,(i-1, i-1)}^{(a)-1} \boldsymbol{\Omega}_{12,(i-1, i-1)}^{(a) \mathrm{H}} \\
& \Delta \boldsymbol{A}_{4}= \\
& -\boldsymbol{T}_{11,(i-1, i-1)}^{-1} \boldsymbol{\Omega}_{12,(i-1, i-1)} \boldsymbol{T}_{22,(i-1, i-1)}^{-1} \boldsymbol{\Omega}_{12,(i-1, i-1)}^{(c) \mathrm{H}}+ \\
& \boldsymbol{T}_{11,(i-1, i-1)}^{-1} \boldsymbol{\Omega}_{12,(i-1, i-1)}^{(c)} . \\
& \frac{\boldsymbol{T}_{22,(i-1, i-1)}^{-1} \boldsymbol{T}_{22,(i-1, i-1)}^{(c)} \boldsymbol{T}_{22,(i-1, i-1)}^{-1}}{1+\boldsymbol{k}_{2,(i-1)}^{(c) \mathrm{H}} \boldsymbol{T}_{22,(i-1, i-1)}^{-1} \boldsymbol{k}_{2,(i-1)}^{c}} \boldsymbol{\Omega}_{12,(i-1, i-1)}^{(a) \mathrm{H}} \\
& \boldsymbol{T}_{11,(i-1, i-1)}^{-1} \boldsymbol{\Omega}_{12,(i-1, i-1)}^{(c)} \boldsymbol{T}_{22,(i-1, i-1)}^{-1} \boldsymbol{\Omega}_{12,(i-1, i-1)}^{(a) \mathrm{H}} \\
& \boldsymbol{T}_{11,(i-1, i-1)}^{-1} \boldsymbol{\Omega}_{12,(i-1, i-1)} . \\
& \frac{\boldsymbol{T}_{22,(i-1, i-1)}^{-1} \boldsymbol{T}_{22,(i-1, i-1)}^{(c)} \boldsymbol{T}_{22,(i-1, i-1)}^{-1}}{1+\boldsymbol{k}_{2,(i-1)}^{(c) \mathrm{H}} \boldsymbol{T}_{22,(i-1, i-1)}^{-1} \boldsymbol{k}_{2,(i-1)}^{(c)}} \boldsymbol{\Omega}_{12,(i-1, i-1)}^{(a) \mathrm{H}} \\
& \Delta B_{3}=\frac{\boldsymbol{T}_{22,(i-1, i-1)}^{(a)-1} \boldsymbol{T}_{22,(i+1, i+1)}^{(c)} \boldsymbol{T}_{22,(i-1, i-1)}^{(a)-1}}{1+\boldsymbol{k}_{2,(i+1)}^{(c) \mathrm{H}} \boldsymbol{T}_{22,(i-1, i-1)}^{(a)-1} \boldsymbol{k}_{2,(i+1)}^{(c)}} . \\
& \boldsymbol{\Omega}_{12,(i-1, i-1)}^{(a)} \boldsymbol{T}_{11,(i-1, i-1)}^{-1} \boldsymbol{\Omega}_{12,(i-1, i-1)}^{(a) \mathrm{H}} \\
& \Delta B_{4}= \\
& -\boldsymbol{T}_{22,(i-1, i-1)}^{-1} \boldsymbol{\Omega}_{12,(i-1, i-1)} \boldsymbol{T}_{11,(i-1, i-1)}^{-1} \boldsymbol{\Omega}_{12,(i-1, i-1)}^{(c) \mathrm{H}}+
\end{aligned}
$$


Zhiyong Suo et al.: Robust PolInSAR optimal interferogram estimation method based on generalized scattering vector

$$
\begin{gathered}
\frac{\boldsymbol{T}_{22,(i-1, i-1)}^{-1} \boldsymbol{T}_{22,(i-1, i-1)}^{(c)} \boldsymbol{T}_{22,(i-1, i-1)}^{-1}}{1+\boldsymbol{k}_{2,(i-1)}^{(c) \mathrm{H}} \boldsymbol{T}_{22,(i-1, i-1)}^{-1} \boldsymbol{k}_{2,(i-1)}^{(c)}} . \\
\boldsymbol{\Omega}_{12,(i-1, i-1)}^{(c)} \boldsymbol{T}_{11,(i-1, i-1)}^{-1} \boldsymbol{\Omega}_{12,(i-1, i-1)}^{(a) \mathrm{H}}- \\
\boldsymbol{T}_{22,(i-1, i-1)}^{-1} \boldsymbol{\Omega}_{12,(i-1, i-1)}^{c} \boldsymbol{T}_{11,(i-1, i-1)}^{-1} \boldsymbol{\Omega}_{12,(i-1, i-1)}^{(a) \mathrm{H}}- \\
\frac{\boldsymbol{T}_{22,(i-1, i-1)}^{-1} \boldsymbol{T}_{22,(i-1, i-1)}^{(c)} \boldsymbol{T}_{22,(i-1, i-1)}^{-1}}{1+\boldsymbol{k}_{2,(i-1)}^{(c) \mathrm{H}} \boldsymbol{T}_{22,(i-1, i-1)}^{-1} \boldsymbol{k}_{2,(i-1)}^{(c)}} . \\
\boldsymbol{\Omega}_{12,(i-1, i-1)} \boldsymbol{T}_{11,(i-1, i-1)}^{-1} \boldsymbol{\Omega}_{12,(i-1, i-1)}^{(a) \mathrm{H}} .
\end{gathered}
$$

Therefore, we have the similar conclusion as (41) and (42).

(iv) Coregistration errors of polarimetric images and interferometric images

When both polarimetric channels and interferometric channels are not accurately coregistered, the scattering vector of pixel $i$ can be expressed as

$$
\begin{gathered}
\boldsymbol{k}_{2,(i)}=\boldsymbol{k}_{2,(i)}^{(a)}+\boldsymbol{k}_{2,(i+1)}^{(c)}+\boldsymbol{k}_{2,(i+1)}^{(b)}= \\
\frac{1}{\sqrt{2}}\left[S_{2 h h,(i)}^{(a)}+S_{2 v v,(i)}^{\left(a_{1}\right)}, S_{2 h h,(i)}^{(a)}-S_{2 v v,(i)}^{\left(a_{1}\right)}, 2 S_{2 h v,(i)}^{\left(a_{2}\right)}\right]^{\mathrm{T}}+ \\
\frac{1}{\sqrt{2}}\left[S_{2 v v,(i+1)}^{\left(c_{1}\right)},-S_{2 v v,(i+1)}^{\left(c_{1}\right)}, 2 S_{2 h v,(i+1)}^{\left(c_{2}\right)}\right]^{\mathrm{T}}+ \\
\frac{1}{\sqrt{2}}\left[S_{2 h h,(i+1)}^{(b)}+S_{2 v v,(i+1)}^{\left(b_{1}\right)}, S_{2 h h,(i+1)}^{(b)}-\right. \\
\left.S_{2 v v,(i+1)}^{\left(b_{1}\right)}, 2 S_{2 h v,(i+1)}^{\left(b_{2}\right)}\right]^{\mathrm{T}} .
\end{gathered}
$$

Then the GSV with coregistration errors is

$$
\begin{gathered}
\boldsymbol{k}_{2}^{(G)}=\left[\boldsymbol{k}_{2,(i-1)}^{(a) \mathrm{T}}+\boldsymbol{k}_{2,(i)}^{(b) \mathrm{T}}+\boldsymbol{k}_{2,(i)}^{(c) \mathrm{T}}, \boldsymbol{k}_{2,(i)}^{(a) \mathrm{T}}+\right. \\
\left.\boldsymbol{k}_{2,(i+1)}^{(b) \mathrm{T}}+\boldsymbol{k}_{2,(i+1)}^{(c) \mathrm{T}}\right]^{\mathrm{T}} .
\end{gathered}
$$

The rearranged GSV is expressed as

$$
\begin{gathered}
\boldsymbol{k}_{2}^{(G)}=\left[\boldsymbol{k}_{2,(i-1)}^{(a) \mathrm{T}}+\boldsymbol{k}_{2,(i+1)}^{(b) \mathrm{T}}+\boldsymbol{k}_{2,(i+1)}^{(c) \mathrm{T}},\right. \\
\left.\boldsymbol{k}_{2,(i)}^{(a) \mathrm{T}}+\boldsymbol{k}_{2,(i)}^{(b) \mathrm{T}}+\boldsymbol{k}_{2,(i)}^{(c) \mathrm{T}}\right]^{\mathrm{T}}= \\
{\left[\boldsymbol{k}_{2,(i-1)}^{(a) \mathrm{T}}+\boldsymbol{k}_{2,(i+1)}^{(b) \mathrm{T}}+\boldsymbol{k}_{2,(i+1)}^{(c) \mathrm{T}}, \boldsymbol{k}_{2,(i)}^{\mathrm{T}}\right]^{\mathrm{T}}=} \\
{\left[\boldsymbol{k}_{2,(i-1)}^{(a) \mathrm{T}}+\boldsymbol{k}_{2,(i+1)}^{(b c) \mathrm{T}}, \boldsymbol{k}_{2,(i)}^{\mathrm{T}}\right]^{\mathrm{T}} .}
\end{gathered}
$$

Therefore, the polarimetric coherence optimal decomposition is executed as

$$
\begin{gathered}
\boldsymbol{T}_{11}^{(G)-1} \boldsymbol{\Omega}_{12}^{(G)} T_{22}^{(G)-1} \boldsymbol{\Omega}_{12}^{(G) \mathrm{H}}= \\
\boldsymbol{I}_{2 \times 2} \otimes \boldsymbol{A}-\left[\begin{array}{cc}
\Delta \boldsymbol{A}_{5} & \mathbf{0} \\
\mathbf{0} & \mathbf{0}
\end{array}\right]+\left[\begin{array}{cc}
\Delta \boldsymbol{A}_{6} & \mathbf{0} \\
\mathbf{0} & \mathbf{0}
\end{array}\right] \\
\boldsymbol{T}_{22}^{(G)-1} \boldsymbol{\Omega}_{12}^{(G) \mathrm{H}} \boldsymbol{T}_{11}^{(G)-1} \boldsymbol{\Omega}_{12}^{(G)}=
\end{gathered}
$$

$$
\boldsymbol{I}_{2 \times 2} \otimes \boldsymbol{B}-\left[\begin{array}{cc}
\Delta \boldsymbol{B}_{5} & \mathbf{0} \\
\mathbf{0} & \mathbf{0}
\end{array}\right]+\left[\begin{array}{cc}
\Delta \boldsymbol{B}_{6} & \mathbf{0} \\
\mathbf{0} & \mathbf{0}
\end{array}\right]
$$

where

$$
\begin{aligned}
& \Delta \boldsymbol{A}_{5}=\frac{\boldsymbol{T}_{22,(i-1, i-1)}^{-1} \boldsymbol{\Omega}_{12,(i-1, i-1)}^{(a)} \boldsymbol{T}_{11,(i-1, i-1)}^{(a)-1} .}{1+\boldsymbol{k}_{1,(i+1)}^{(b c) \mathrm{H}} T_{11,(i-1, i-1)}^{(a)-1} \boldsymbol{k}_{1,(i+1)}^{(b c)}} . \\
& \boldsymbol{T}_{11,(i+1, i+1)}^{(b c)} \boldsymbol{T}_{11,(i-1, i-1)}^{(a)-1} \boldsymbol{\Omega}_{12,(i-1, i-1)}^{(a) \mathrm{H}} \\
& \Delta \boldsymbol{A}_{6}= \\
& -\boldsymbol{T}_{11,(i-1, i-1)}^{-1} \boldsymbol{\Omega}_{12,(i-1, i-1)} \boldsymbol{T}_{22,(i-1, i-1)}^{-1} \boldsymbol{\Omega}_{12,(i-1, i-1)}^{(b c) \mathrm{H}}+ \\
& \boldsymbol{T}_{11,(i-1, i-1)}^{-1} \boldsymbol{\Omega}_{12,(i-1, i-1)}^{(b c)} . \\
& \frac{\boldsymbol{T}_{22,(i-1, i-1)}^{-1} \boldsymbol{T}_{22,(i-1, i-1)}^{(b c)} \boldsymbol{T}_{22,(i-1, i-1)}^{-1}}{1+\boldsymbol{k}_{2,(i-1)}^{(b c) \mathrm{H}} \boldsymbol{T}_{22,(i-1, i-1)}^{-1} \boldsymbol{k}_{2,(i-1)}^{b c}} \boldsymbol{\Omega}_{12,(i-1, i-1)}^{(a) \mathrm{H}} \\
& \boldsymbol{T}_{11,(i-1, i-1)}^{-1} \boldsymbol{\Omega}_{12,(i-1, i-1)}^{(b c)} \boldsymbol{T}_{22,(i-1, i-1)}^{-1} \boldsymbol{\Omega}_{12,(i-1, i-1)}^{(a) \mathrm{H}} \\
& \boldsymbol{T}_{11,(i-1, i-1)}^{-1} \boldsymbol{\Omega}_{12,(i-1, i-1)} . \\
& \frac{\boldsymbol{T}_{22,(i-1, i-1)}^{-1} \boldsymbol{T}_{22,(i-1, i-1)}^{(b c)} \boldsymbol{T}_{22,(i-1, i-1)}^{-1}}{1+\boldsymbol{k}_{2,(i-1)}^{(b c) \mathrm{H}} \boldsymbol{T}_{22,(i-1, i-1)}^{-1} \boldsymbol{k}_{2,(i-1)}^{(b c)}} \boldsymbol{\Omega}_{12,(i-1, i-1)}^{(a) \mathrm{H}} \\
& \Delta \boldsymbol{B}_{5}=\frac{\boldsymbol{T}_{22,(i-1, i-1)}^{(a)-1} \boldsymbol{T}_{22,(i+1, i+1)}^{(b c)} \boldsymbol{T}_{22,(i-1, i-1)}^{(a)-1}}{1+\boldsymbol{k}_{2,(i+1)}^{(b c) \mathrm{H}} \boldsymbol{T}_{22,(i-1, i-1)}^{(a)-1} \boldsymbol{k}_{2,(i+1)}^{(b c)}} . \\
& \boldsymbol{\Omega}_{12,(i-1, i-1)}^{(a)} \boldsymbol{T}_{11,(i-1, i-1)}^{-1} \boldsymbol{\Omega}_{12,(i-1, i-1)}^{(a) \mathrm{H}} \\
& \Delta \boldsymbol{B}_{6}= \\
& -\boldsymbol{T}_{22,(i-1, i-1)}^{-1} \boldsymbol{\Omega}_{12,(i-1, i-1)} \boldsymbol{T}_{11,(i-1, i-1)}^{-1} \boldsymbol{\Omega}_{12,(i-1, i-1)}^{(b c) \mathrm{H}}+ \\
& \frac{\boldsymbol{T}_{22,(i-1, i-1)}^{-1} \boldsymbol{T}_{22,(i-1, i-1)}^{(b c)} \boldsymbol{T}_{22,(i-1, i-1)}^{-1}}{1+\boldsymbol{k}_{2,(i-1)}^{(b c) \mathrm{H}} \boldsymbol{T}_{22,(i-1, i-1)}^{-1} \boldsymbol{k}_{2,(i-1)}^{(b c)}} . \\
& \boldsymbol{\Omega}_{12,(i-1, i-1)}^{(b c)} \boldsymbol{T}_{11,(i-1, i-1)}^{-1} \boldsymbol{\Omega}_{12,(i-1, i-1)}^{(a) \mathrm{H}} \\
& \boldsymbol{T}_{22,(i-1, i-1)}^{-1} \boldsymbol{\Omega}_{12,(i-1, i-1)}^{(b c)} \boldsymbol{T}_{11,(i-1, i-1)}^{-1} \boldsymbol{\Omega}_{12,(i-1, i-1)}^{(a) \mathrm{H}} \\
& \frac{\boldsymbol{T}_{22,(i-1, i-1)}^{-1} \boldsymbol{T}_{22,(i-1, i-1)}^{(b c)} \boldsymbol{T}_{22,(i-1, i-1)}^{-1}}{1+\boldsymbol{k}_{2,(i-1)}^{(b c) \mathrm{H}} \boldsymbol{T}_{22,(i-1, i-1)}^{-1} \boldsymbol{k}_{2,(i-1)}^{(b c)}} . \\
& \boldsymbol{\Omega}_{12,(i-1, i-1)} \boldsymbol{T}_{11,(i-1, i-1)}^{-1} \boldsymbol{\Omega}_{12,(i-1, i-1)}^{(a) \mathrm{H}} .
\end{aligned}
$$

Therefore, we have the similar conclusion as (41) and (42) again.

(v) Unknown coregistration errors

In practice, the coregistration errors between the interferometric SAR images and/or the polarimetric SAR images are often unknown. In previous disscussion, we assume that the coregistration errors are only in the slave antenna, but the coregistration errors of the polarimetric SAR images of master antenna is unavoidable. Considering that the coregistration errors are random, and the error direction and error magnitude are also unknown, the GSV 
should be constructed by using the scattering vectors of its neighboring eight pixels. In the case, the generalized coherency matrix $\boldsymbol{T}_{11}^{(G)}, \boldsymbol{T}_{22}^{(G)}$ and the cross interferometric matrix $\Omega_{12}^{(G)}$ are all $27 \times 27$. The number of eigenvalues of the coefficient matrix is 27 . Without coregsitration errors, there are three different eigenvalues and each eigenvalue is a nine-fold eigenvalue.

Due to the influence of noise and coregistration errors, the Kronecker product of the identity matrix $\boldsymbol{I}$ and $\boldsymbol{A}$ (or $\boldsymbol{B})$ does not hold strictly, so the optimal scattering mechanisms (or interferograms) are estimated by the combination of weighted projection of $\boldsymbol{\Omega}_{12}^{(G)}$ onto different eigenvectors.

Supposing the eigenvalues of the GSV-based coefficient matrix are $\lambda_{1} \geqslant \cdots \geqslant \lambda_{9}>\lambda_{10} \geqslant \lambda_{11} \cdots \geqslant \lambda_{18}>$ $\lambda_{19} \geqslant \lambda_{20} \cdots \geqslant \lambda_{27}$, and the corresponding eigenvectors are $\boldsymbol{\omega}_{1}^{(G)}, \boldsymbol{\omega}_{2}^{(G)}, \cdots, \boldsymbol{\omega}_{26}^{(G)}, \boldsymbol{\omega}_{27}^{(G)}$, the optimal scattering mechanisms can be expressed as

$$
\begin{gathered}
\Phi_{\text {opt } 1}=\arg \left(\sum_{i=1}^{9} \lambda_{i} \boldsymbol{\omega}_{i}^{(G) \mathrm{H}} \boldsymbol{\Omega}_{12}^{(G)} \omega_{i}^{(G)} / \sum_{i=1}^{9} \lambda_{i}\right) \\
\Phi_{\text {opt } 2}=\arg \left(\sum_{i=10}^{18} \lambda_{i} \boldsymbol{\omega}_{i}^{(G) \mathrm{H}} \boldsymbol{\Omega}_{12}^{(G)} \boldsymbol{\omega}_{i}^{(G)} / \sum_{i=10}^{18} \lambda_{i}\right) \\
\Phi_{\text {opt } 3}=\arg \left(\sum_{i=19}^{27} \lambda_{i} \boldsymbol{\omega}_{i}^{(G) \mathrm{H}} \boldsymbol{\Omega}_{12}^{(G)} \boldsymbol{\omega}_{i}^{(G)} / \sum_{i=19}^{27} \lambda_{i}\right) .
\end{gathered}
$$

(vi) Noise influence analysis

The noise influence can be divided into two parts, including the SAR images' noise and the non-coregistered signals. The SAR images' noise distributes in the whole frequency domain. However, the SAR images' noise can be considered as complex circular Gauss noise [22], it can be filtered by eigen-decomposition and subspace projection as (63)-(65). Therefore, the SAR images' noise will not affect optimal interferogram estimation.

When the SAR images are not accurately coregistered, the GSV-based coefficient matrix will be affected by the non-coregistered signals. The non-coregistered signals' power is far less than the coregistered signals' power. In [22], the authors analyze the effect of the non-coregistered signals on InSAR phase estimation. The non-coregistered signals will increase the dimension of signal subspace, however, they do not affect the final estimate results of interferogram. The determination of signal subspace dimension is discussed in detail [24]. Because the total power of coregistration errors is much lower than the coregistered parts, if we use (63)-(65) to estimate the optimal interferograms, a phase bias will be introduced to the final estimates, and the phase bias is constant for the whole interferogram, i.e., it is a systematic error for the estimated interferogram. Therefore, the phase bias can be calibrated with auxiliary data, like the corner reflector and/or the bare soil, etc.

\section{Procedures of optimal scattering mechanisms estimation using GSV}

In this section, we give the detailed procedures to estimate the optimal scattering mechamisms (or interferograms) based on GSV.

Step 1 SAR images coregistration

We use the span image or the corresponding polarimetric SAR image to coregister the PolSAR images. The main object of Step 1 is to coarsely coregister the interferometric SAR images by cross correlation or other techniques for generalized scattering vector construction.

Step 2 Generalized scattering vector construction

The GSV is constructed by the Pauli scattering vector of the processing pixel and its neighboring pixels. The GSV must be constructed in the interferometric master channel and the interferometric slave channel respectively.

Step 3 Generalized coherency matrix $\boldsymbol{T}_{11}^{(G)}, \boldsymbol{T}_{22}^{(G)}$ and interferometric matrix $\boldsymbol{\Omega}_{12}^{(G)}$ estimation

Generally, we cannot get the statistical expectation of $\boldsymbol{T}_{11}^{(G)}, \boldsymbol{T}_{22}^{(G)}$ and $\boldsymbol{\Omega}_{12}^{(G)}$, so we estimate them using i.i.d samples. Supposing the GSVs are i.i.d samples, we can estimate $\boldsymbol{T}_{11}^{(G)}, \boldsymbol{T}_{22}^{(G)}$ and $\boldsymbol{\Omega}_{12}^{(G)}$ as

$$
\begin{aligned}
& \boldsymbol{T}_{11}^{(G)}=\frac{1}{2 N+1} \sum_{n=-N}^{N} \boldsymbol{k}_{1}^{(G)}(i+n) \boldsymbol{k}_{1}^{(G) \mathrm{H}}(i+n) \\
& \boldsymbol{T}_{22}^{(G)}=\frac{1}{2 N+1} \sum_{n=-N}^{N} \boldsymbol{k}_{2}^{(G)}(i+n) \boldsymbol{k}_{2}^{(G) \mathrm{H}}(i+n) \\
& \boldsymbol{\Omega}_{12}^{(G)}=\frac{1}{2 N+1} \sum_{n=-N}^{N} \boldsymbol{k}_{1}^{(G)}(i+n) \boldsymbol{k}_{2}^{(G) \mathrm{H}}(i+n)
\end{aligned}
$$

where $2 N+1$ is the i.i.d sample number.

Step 4 Coefficient matrix eigen-composition

Step 5 Eigenvalues sorting and corresponding eigenvectors sequence adjusting

The eigenvalues are sorted in the decreasing order, meanwhile the sequence of eigenvectors are adjusted in order to correspond to their eigenvalue respectively.

Step 6 Optimal interferograms (or scattering mechanisms) estimation using (63) - (65).

\section{Experiment results and performance investigation}

In this section, the performance of two different methods, i.e., the conventional Pauli-based optimal scattering 
mechanisms estimation method and the GSV-based optimal scattering mechanisms estimation method, are investigated by using the simulated data and the actual PALSAR data.

\subsection{PolSARPro simulated data investigation}

We use the simulated PolInSAR data generated by PolSARPro to investigate the performance of the optimal interferograms estimation based on the Pauli scattering vector and the GSV respectively. The simulation parameters are listed in Table 1.

Table 1 PolSARPro simulation parameters

\begin{tabular}{cc}
\hline Simulation parameter & Value \\
\hline Antennae number & 2 \\
Master antenna slant range /m & 4242.6406 \\
Master antenna incidence angle /( ) & 45 \\
Slave antenna slant range /m & 4250.4236 \\
Slave antenna incidence angle /( ) & 45.0858 \\
Center frequency /GHz & 1.3 \\
Azimuth resolution /m & 1.5 \\
Range resolution /m & 1.0607 \\
Mean tree height /m & 10.0 \\
Tree density per hectare & 900 \\
Ground slope in azimuth direction & 0.02 \\
Ground slope in ground range direction & 0.01 \\
\hline
\end{tabular}

In the following, we analyze the performance of the GSV-based method with different coregistration errors. The Pauli-based conventional optimal interferogram estimation results are also given for comparison. Four cases are analyzed in the following section.

Case 1 Accurate coregistration

We assume that $S_{1, h h}, S_{1, v v}$ and $S_{1, h v}$ are accurately coregistered, and $S_{2, h h}, S_{2, v v}$ and $S_{2, h v}$ are also accurately coregistered. Futhermore, we assume that interferometric SAR images pairs $S_{1, h h}$ and $S_{2, h h}$ are also accurately coregistered. Under the assumptions above, all the SAR images are accurately coregistered.

Case 2 Coregistration errors of $1 / 10$ pixels between interferometric SAR images

For this case, we assume that there are no coregistration errors between polarimetric SAR images, i.e., $S_{1, h h}, S_{1, v v}$ and $S_{1, h v}$ are accurately coregistered, and $S_{2, h h}, S_{2, v v}$ and $S_{2, h v}$ are also accurately coregistered. Without loss of generality, taking $S_{1, h h}$ as a reference image, we set $1 / 10$ pixels coregistration errors between $S_{1, h h}$ and $S_{2, h h}$ in range direction. Therefore, $S_{1, v v}$ and $S_{2, v v}$, as well as $S_{1, h v}$ and $S_{2, h v}$, also have $1 / 10$ pixels coregistration errors in the same range direction.

Case 3 Coregistration errors of $1 / 10$ pixels between polarimetric SAR images

For this case, we assume that there are no coregistration errors in interferometric SAR image pairs, i.e., $S_{1, h h}$ and
$S_{2, h h}$ are also accurately coregistered. However, there are coregistration errors between polarimetric SAR images. Taking $S_{m, h h}(m=1,2)$ as reference images, we set $1 / 10$ pixels coregistration errors between $S_{m, h v}$ and $S_{m, h h}$ in the same range direction.

Case 4 Coregistration errors of $1 / 10$ pixels in both polarimetric SAR images and interferometric SAR images

Taking $S_{m, h h}(m=1,2)$ as the reference images, we first set image coregistration errors of $1 / 10$ pixels between $S_{m, h v}$ and $S_{m, h h}$ in range direction, and set image coregistration errors of 1/10 pixels between $S_{m, v v}$ and $S_{m, h h}$ in azimuth direction. Futhermore, taking $S_{1, h h}, S_{1, h v}$ and $S_{1, v v}$ as reference images, we set $1 / 10$ pixels coregistration errors between the corresponding polarimetric SAR images (i.e., $S_{2, h h}, S_{2, h v}$ and $S_{2, v v}$ ) in range direction, respectively. In this case, the coregistration errors exist not only between polarimetric SAR images but also between interferometric SAR images.

The optimal scattering mechanisms (i.e., the optimal interferograms) with different errors are shown in Fig. 4Fig. 7.

Fig. 4 shows results of Case 1, where Fig. 4(a)Fig. 4(c) are the interferograms obtained by the Paulibased method, and Fig. 4(d)-Fig. 4(f) are the interferograms obtained by the GSV-based method. From Fig. 4, we can see that when all the SAR images are coregistered accurately, the optimal interferograms can be estimated well either the Pauli-based method or the GSV-based method is used. However, the phase fluctuations of Fig. 4(d)Fig. 4(f) are smaller than those of Fig. 4(a)-Fig. 4(c), especially in the area where the trees are placed.

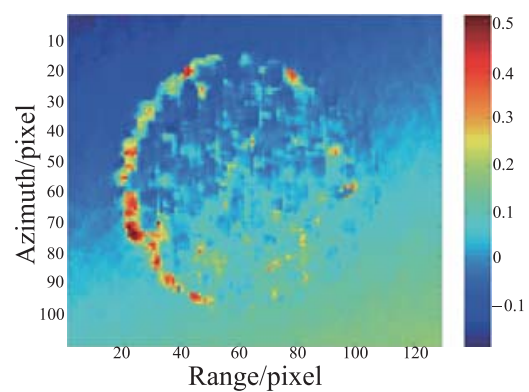

(a) Pauli-based optimal interferogram of the largest eigenvalue

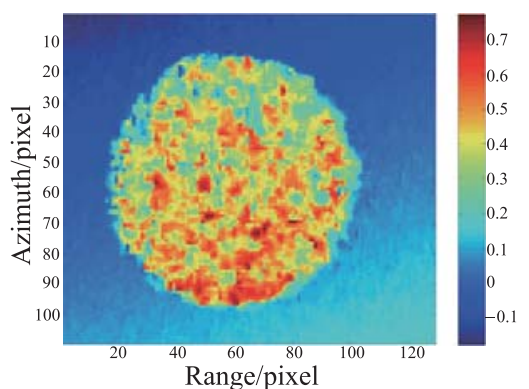

(b) Pauli-based optimal interferogram of the second largest eigenvalue 


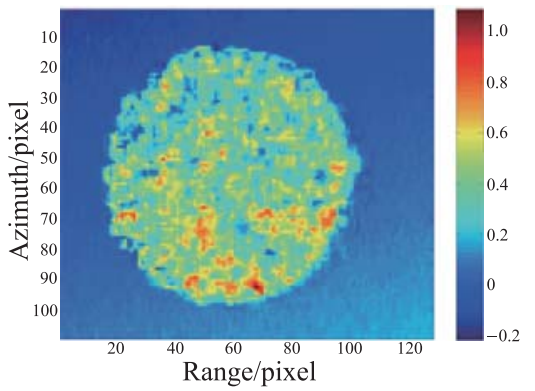

(c) Pauli-based optimal interferogram of the smallest eigenvalue

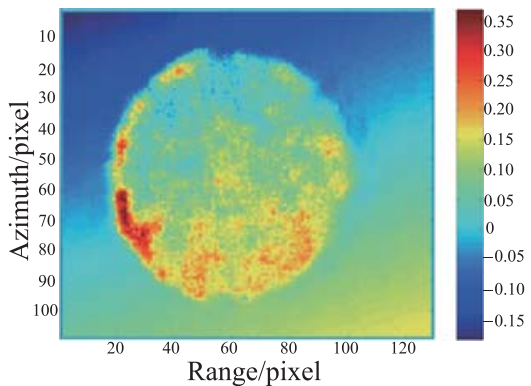

(d) GSV-based optimal interferogram of the largest eigenvalue

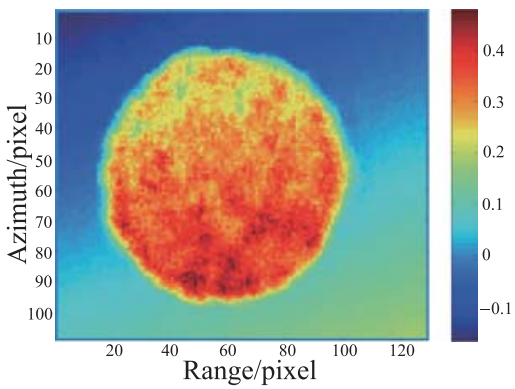

(e) GSV-based optimal interferogram of the second largest eigenvalue

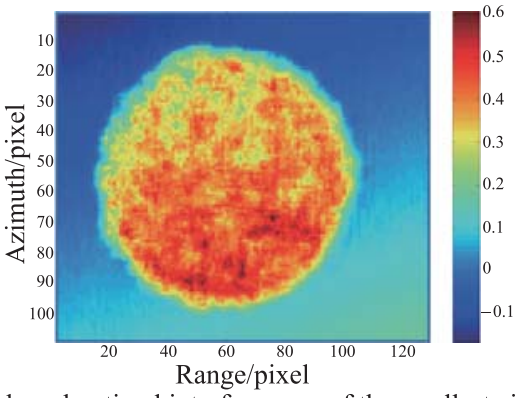

(f) GSV-based optimal interferogram of the smallest eigenvalue

Fig. 4 Optimal interferograms of Case 1

Fig. 5 shows the estimated results of Case 2, where Fig. 5(a)-Fig. 5(c) are the optimal interferograms obtained by the Pauli-based method, and Fig. 5(d) - Fig. 5(f) are the optimal interferograms obtained by the GSV-based method. From Fig. 5, we can see that when there are coregistration errors between interferometric SAR images, the phase fluctuations of Fig. 5(a)-Fig. 5(c) are much worse than those in Fig. 4(a)-Fig. 4(c), respectively. However, the estimated results of Fig. 5(d) - Fig. 5(f) are slightly deteriorated though there are coregistration errors between interferometric SAR images.

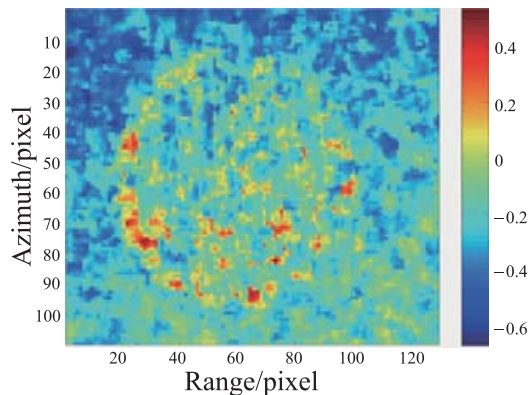

(a) Pauli-based optimal interferogram of the largest eigenvalue

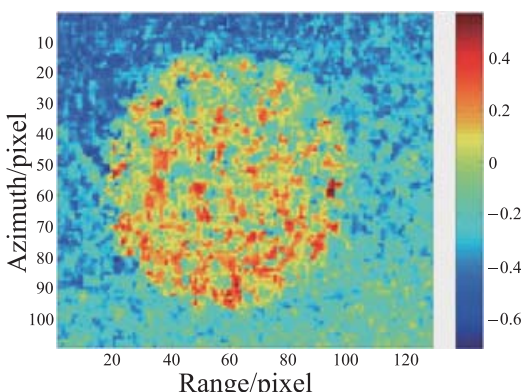

(b) Pauli-based optimal interferogram of the second largest eigenvalue

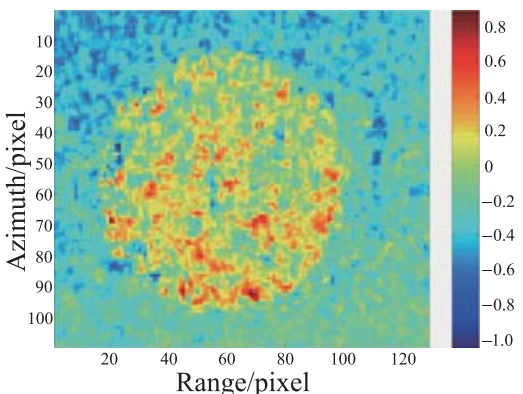

(c) Pauli-based optimal interferogram of the smallest eigenvalue

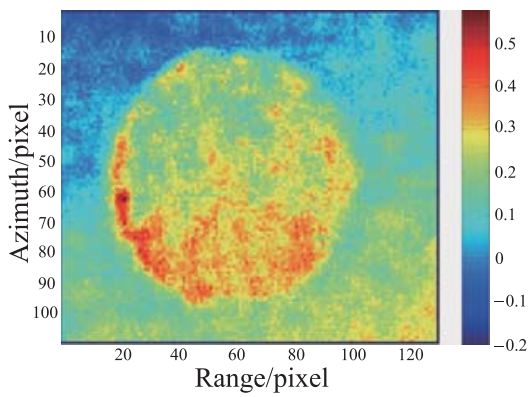

(d) GSV-based optimal interferogram of the largest eigenvalue

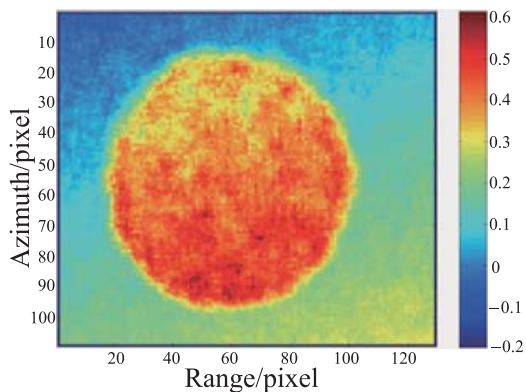

(e) GSV-based optimal interferogram of the second largest eigenvalue 


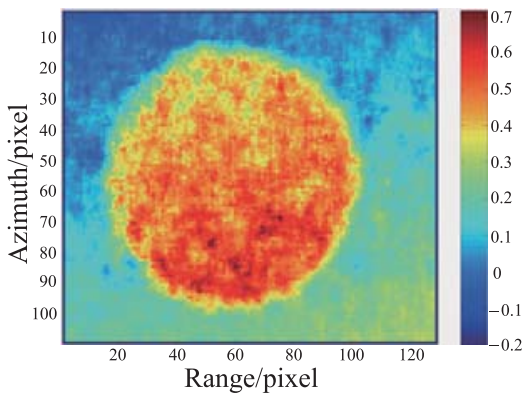

(f) GSV-based optimal interferogram of the smallest eigenvalue

Fig. 5 Optimal interferograms of Case 2

The optimal interferograms of Case 3 are shown in Fig. 6, where Fig. 6(a)-Fig. 6(c) are the optimal interferograms obtained by the Pauli-based method, and Fig. 6(d) - Fig. 6(f) are the optimal interferograms obtained by the GSV-based method. From Fig. 6(d)-Fig. 6(f), we can draw the similar conclusion that coregistration errors between polarimetric SAR images have almost no effect on the optimal interferograms estimation while the GSVbased method is used. Generally, for the single polarimetric InSAR processing, the coregistration errors of $1 / 10$ pixels are enough for DEM generation. However, from Fig. 6, we can see that the needed coregistration accuracy for PolInSAR processing is higher than the single polarimetric InSAR processing.

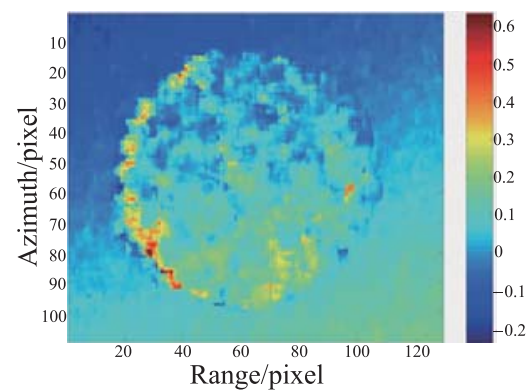

(a) Pauli-based optimal interferogram of the largest eigenvalue

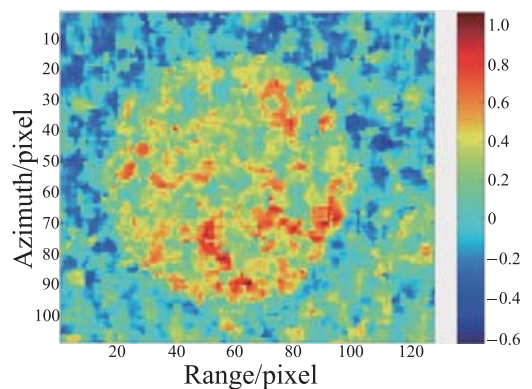

(b) Pauli-based optimal interferogram of the second largest eigenvalue

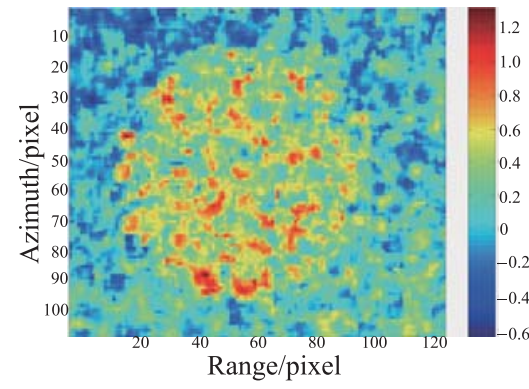

(c) Pauli-based optimal interferogram of the smallest eigenvalue

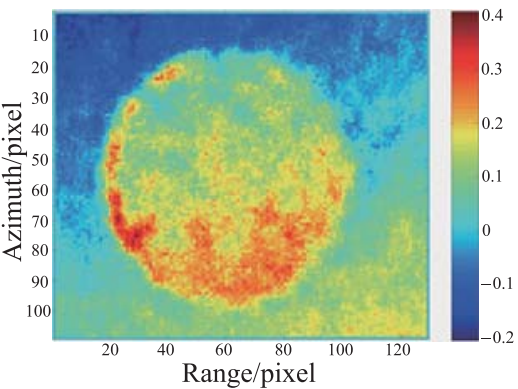

(d) GSV-based optimal interferogram of the largest eigenvalue

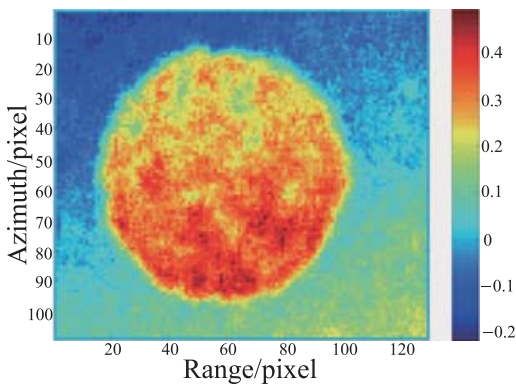

(e) GSV-based optimal interferogram of the second largest eigenvalue

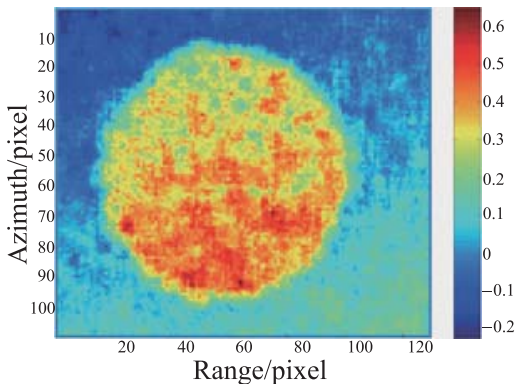

(f) GSV-based optimal interferogram of the smallest eigenvalue

Fig. 6 Optimal interferograms of Case 3

The optimal interferograms of Case 4 are shown in Fig. 7, where Fig. 7(a)-Fig. 7(c) are the optimal interferograms obtained by the Pauli-based method, and Fig. 7(d)-Fig. 7(f) are the optimal interferograms obtained by the GSV-based method. The estimated results of Fig. 7(d) - Fig. 7(f) show that the GSV-based method has a changeless performance compared to Fig. 4(d) - Fig. 4(f), where there are no coregistration errors between SAR images. 


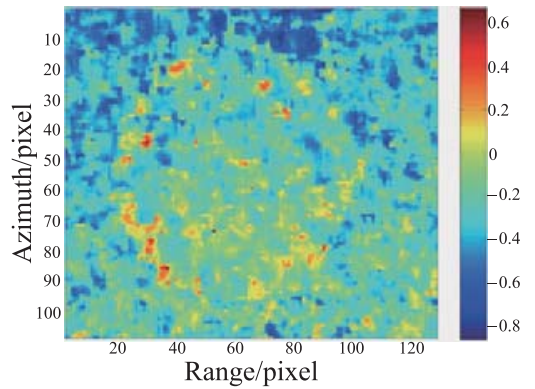

(a) Pauli-based optimal interferogram of the largest eigenvalue

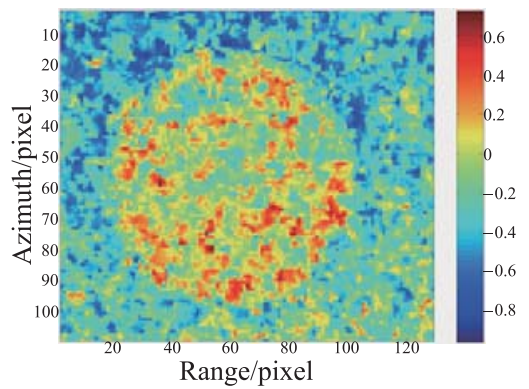

(b) Pauli-based optimal interferogram of the second largest eigenvalue

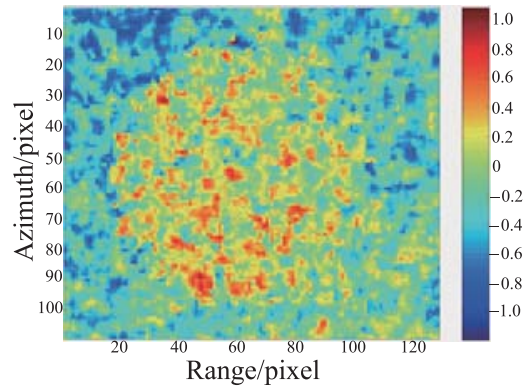

(c) Pauli-based optimal interferogram of the smallest eigenvalue

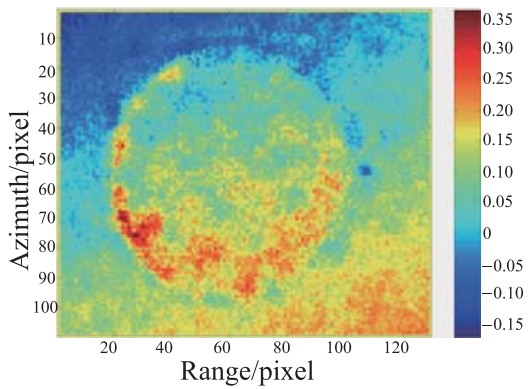

(d) GSV-based optimal interferogram of the largest eigenvalue

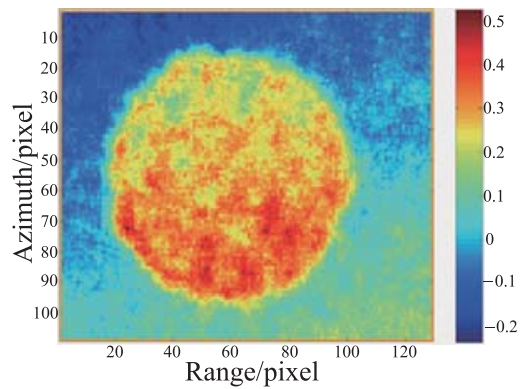

(e) GSV-based optimal interferogram of the second largest eigenvalue

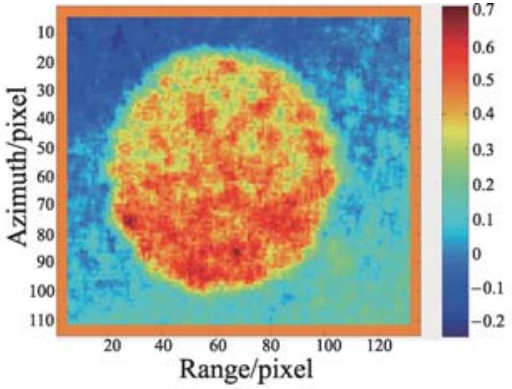

(f) GSV-based optimal interferogram of the smallest eigenvalue

Fig. 7 Optimal interferograms of Case 4

Comparing Fig. 4(d)-Fig. 4(f), Fig. 5(d)-Fig. 5(f), Fig. 6(d) - Fig. 6(f), and Fig. 7(d) - Fig. 7(f), we can conclude that the GSV-based method is robust to SAR images coregistration errors, including the polarimetric SAR images coregistration errors and/or the interferometric SAR images coregistration errors. On the contrary, the conventional Pauli-based method is greatly affected by the coregistration errors. However, from the analysis in Section 3, we know that the proposed method have more computational burden than the Pauli-based method because the eigendecompostion of high-dimension matrix is necessary. In order to reduce the computational complexity, we can use the scattering vectors of four adjacent pixels around the processing pixel to construct the GSV. The relative position of the pixels likes the symbol "+".

From Fig. 4-Fig. 7, we can see that the Pauli-based method failes to estimate the optimal scattering mechanisms when there are coregistration errors. However, the GSV-based method is robust to coregistration errors and has a smaller phase fluctuations than the Pauli-based method when there are coregistration errors.

\subsection{Real PALSAR data}

To confirm the effectiveness of the proposed method, we use the PALSAR data to investigate the interferograms estimation performance with the GSV-based method and the Pauli-based method in this section. The two datasets used for PolInSAR processing are acquired in March 13, 2007 (Site ID ALPSRP060247100) and April 28, 2007 (Site ID ALPSRP066957100). The illuminated area is the Amazon River in Brazil.

For the PALSAR data processing, the first thing to do is to coregister the PolInSAR images. Generally speaking, the polarimetric SAR images are coregistered with high accuracy when the datasets are released. Therefore, we should coregister the interferometric SAR images in order to excute PolInSAR processing. We first select a reference image pair with $S_{1, h h}$ and $S_{2, h h}$ to obtain the coregistration offsets of $S_{2, h h}$ relative to $S_{1, h h}$, then the 
coregistration offsets are also used to $S_{2, h v}$ and $S_{2, v v}$. The optimal interferograms are given in Fig. 8. We can see that the phase noises in Fig. 8(d)-Fig. 8(f) are smaller than those in Fig. 8(a)-Fig. 8(c), especially in the third scattering mechnism of Fig. 8(c) and Fig. 8(f). Therefore, the GSV-based method has a better performance than the Pauli-based method in the optimal interferograms estimation.

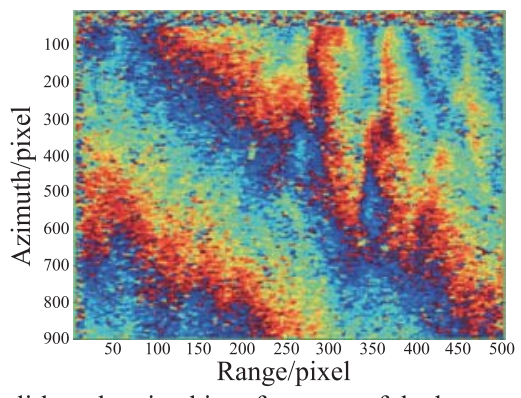

(a) Pauli-based optimal interferogram of the largest eigenvalue

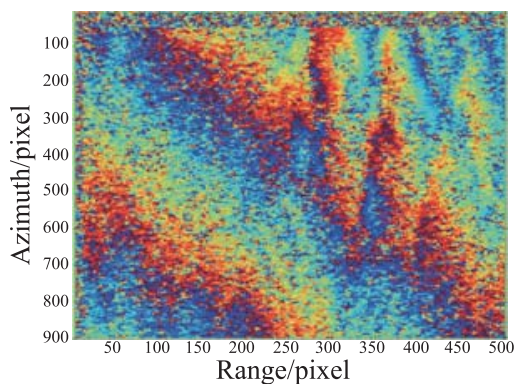

(b) Pauli-based optimal interferogram of the second largest eigenvalue

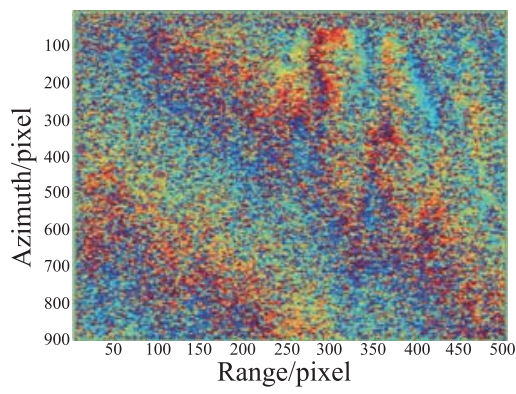

(c) Pauli-based optimal interferogram of the smallest eigenvalue

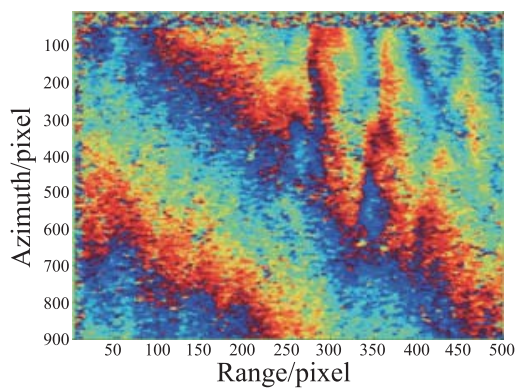

(d) GSV-based optimal interferogram of the largest eigenvalue

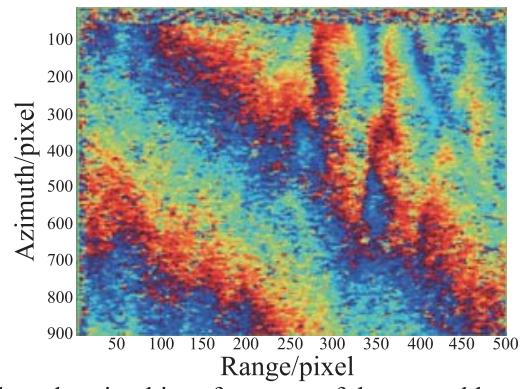

(e) GSV-based optimal interferogram of the second largest eigenvalue

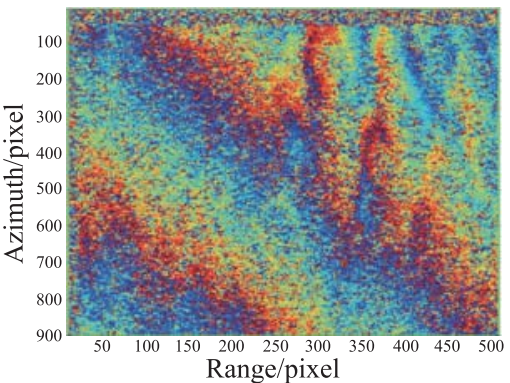

(f) GSV-based optimal interferogram of the smallest eigenvalue

Fig. 8 Optimal interferograms of PALSAR data

To further investigate the performance of the GSV-based method and the Pauli-based method, we count the residue numbers in different scattering mechanisms. The residue numbers are shown in Table 2. From Table 2, we can see that for the corresponding scattering mechanisms, the residue numbers of the GSV-based method are smaller than those of the Pauli-based method. The residue numbers of the GSV-based method are reduced by $8.1 \%, 53.27 \%$ and $28.1 \%$ respectively compared to the optimal interferogram of Fig. 8(a)-Fig. 8(c) obtained by the Pauli-based method. The results show that the GSV-based method has a better estimation performance than the Pauli-based method.

Table 2 Residue numbers of GSV-based method and Pauli-based method for different scattering mechanisms

\begin{tabular}{lc}
\hline Different scattering mechanisms & Residue number \\
\hline Pauli-based method for Fig. 8(a) & 3917 \\
Pauli-based method for Fig. 8(b) & 13376 \\
Pauli-based method for Fig. 8(c) & 39468 \\
GSV-based method for Fig. 8(d) & 3601 \\
GSV-based method for Fig. 8(e) & 6250 \\
GSV-based method for Fig. 8(f) & 28398 \\
\hline
\end{tabular}

\subsection{Computational complexity analysis}

Assuming the SAR images are coarsely coregistered, the computational complexity is mainly determined by Step 3, Step 4 and Step 6. For Step 3, the computational complexity is $O\left(M^{3}\right)$, where $M$ is the dimension of the covariance matrix. For Step 4, the computational complexity of eigen decomposition is $O\left(M^{3}\right)$. For Step 6, the 
computational complexity is $O\left(M^{2}\right)$. We can see that for the larger dimension $M$, the more computational load is needed. Therefore, the computational complexity of the GSV-based method is larger than the conventional Paulibased method. In Section 5.2, the relative position of the pixels as the symbol "+" are suggested to reduce the computational complexity.

\section{Conclusions}

In this paper, we propose a new model, GSV, to estimate the optimal scattering mechanisms. Compared to the conventional Pauli-based optimal interferograms estimation method, the proposed GSV-based method can obtain a more robust estimate when there are coregistration errors between polarimetric SAR images and /or interferometric SAR images. From the construction of the GSV, we can see that the optimal scattering mechanisms will be recovered adaptively even with images coregistration errors. When the GSV is used, the dimension of the generalized coherency matrix will be greatly increased, therefore, the defect of the proposed method has an exhausted computational burden. Ideally, there are three different eigenvalues, and each eigenvalue is nine-fold. However, the eigenvalues are all different because that the coregistration errors are inevitable in practice. Therefore, the eigenvalueweighted method is used to estimate the optimal interferograms when the GSV-based method is performed. Finally, we use the repeated pass PALSAR data to verify the effectiveness of the GSV-based method.

\section{References}

[1] K. P. Papathanassiou, S. R. Cloude. Single-baseline polarimetric SAR interferometry. IEEE Trans. on Geoscience and Remote Sensing, 2001, 39(11): 2352-2363.

[2] R. N. Treuhaft, S. R. Cloude. The structure of oriented vegetation from polarimetric interferometry. IEEE Trans. on Geoscience and Remote Sensing, 1999, 37(5): 2620-2624.

[3] R. N. Treuhaft, P. R. Siqueira. Vertical structure of vegetated land surfaces from interferometric and polarimetric radar. $\mathrm{Ra}$ dio Science, 2000, 35(1): 141-177.

[4] K. P. Papathanassiou, S. R. Cloude. Polarimetric effects in repeat-pass SAR interferometry. Proc. of the International Geoscience and Remote Sensing Symposium, 1997: 19261928.

[5] S. R. Cloude. Robust parameter estimation using dual baseline polarimetric SAR interferometry. Proc. of the International Geoscience and Remote Sensing Symposium, 2002: 838 - 840.

[6] J. J. Van Zyl. Unsupervised classification of scattering behavior using radar polarimetry data. IEEE Trans. on Geoscience and Remote Sensing, 1989, 27(1): 36-45.

[7] J. S. Lee, M. R. Grunes, T. L. Ainsworth, et al. Unsupervised classification using polarimetric decomposition and the complex wishart classifier. IEEE Trans. on Geoscience and Remote Sensing, 1999, 37(5): 2249-2258.

[8] L. F. Famil, E. Pottier, J. S. Lee. Unsupervised classification of multifrequency and fully polarimetric SAR images based on the H/A/Alpha - wishart classifier. IEEE Trans. on Geoscience and Remote Sensing, 2001, 39(11): 2332-2342.

[9] B. Zitova, J. Flusser. Image registration methods: a survey. Image and Vision Computing, 2003, 21(11): 977-1000.

[10] R. Scheiber, A. Moreira. Coregistration of SAR images using interferometric spectral diversity. IEEE Trans. on Geoscience and Remote Sensing, 2000, 38(5): 2179-2191.

[11] Q. Lin, J. Vesecky, H. Zebker. New approaches in interferometric SAR data processing. IEEE Trans. on Geoscience and Remote Sensing, 1992, 30(3): 560-567.

[12] A. K. Gabriel, R. M. Goldstein. Crossed orbit interferometry: theory and experiment results from SIR-B. International Journal of Remote Sensing, 1988, 9(5): 857-872.

[13] J. Yang, Y. Peng, S. Lin. Similarity between two scattering matrices. Electronics Letters, 2001, 37(3): 193 - 194.

[14] L. Tan, R. Yang. Investigation on coregistration of polarimetric interferometry data with Cameron decomposition. Systems Engineering and Electronics, 2009, 31(6): $1284-1287$. (in Chinese)

[15] W. L. Cameron, L. K. Leung. Feature motivated polarization scattering matrix decomposition. Proc. of the IEEE International Radar Conference, 1990: 549-557.

[16] W. L. Cameron, N. N. Youssef, L. K. Leung. Simulated polarimetric signatures of primitive geometrical shapes. IEEE Trans. on Geoscience and Remote Sensing, 1996, 34(3): 793 - 803.

[17] J. Liu, X. Yu. Research on SAR image matching technology based on SIFT. The International Achieves of the Photogrammetry, Remote Sensing and Spatial Information Sciences, 2008, 15(B1): 403-408.

[18] R. A. Horn, C. R. Johnson. Matrix analysis. Cambridge: Cambridge University Press, 1985.

[19] Z. Li, Z. Bao, Z. Suo. A joint image coregistration, phase noise suppression and phase unwrapping method based on subspace projection for multibaseline InSAR systems. IEEE Trans. on Geoscience and Remote Sensing, 2007, 45(3): 584-591.

[20] Z. Suo, Z. Li, Z. Bao. A multi-channel SAR-GMTI method robust to coregistration error of SAR images. IEEE Trans. on Aerospace and Electronics System, 2010, 46(4): 2035 - 2043.

[21] S. R. Cloude, K. P. Papathanassiou. Polarimetric optimisation in radar interferometry. Electronics Letters, 1997, 33(13): $1176-1178$.

[22] Z. Li, Z. Bao, H. Li, et al. Image autocoregistration and InSAR interferogram estimation using joint subspace projection. IEEE Trans. on Geoscience and Remote Sensing, 2006, 44(2): $288-297$.

[23] J. Sherman, W. J. Morrison. Adjustment of an inverse matrix corresponding to changes in one element of a given matrix. Annals of Mathematical Statistics, 1950, 21: 124-127.

[24] H. Li. Study on key techniques of signal processing for interferometric synthetic aperture radar. Xi' an: Xidian University, 2008.

\section{Biographies}

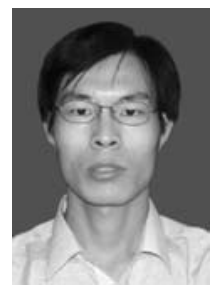

Zhiyong Suo was born in 1979. He received his B.S. degree, M.S. degree and Ph.D degree in electronic engineering in 2003, 2006 and 2009 respectively, from Xidian University, Xi'an, China. Now he is an associate professor in National Lab of Radar Signal Processing, Xidian University. His main research interests are SAR, InSAR, PolInSAR and DInSAR processing. E-mail: zysuo@xidian.edu.cn 


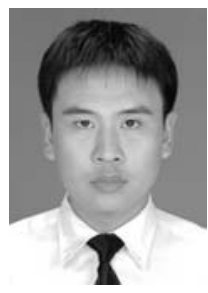

Ming $\mathbf{L i}$ was born in 1983. He received his B.S. and M.S. degrees in 2001 and 2005 respectively in electronic science and technology from Aviation Engineering Insititutes of Nanchang, signal and information processing from Xi' an Electronic and Engineering University. He is currently an engineer at Beijing Aerospace Automatic Control Institute and National Key Laboratory of Science and Technology on Aerospace Intelligent Control, Beijing, China. His research interests are radar terminal guidance system design, radar aided navigation system design, and radar signal processing.

E-mail: liming_up@163.com 